\title{
L'HOMME L'Homme
}

192 | 2009

\section{Ecritures et langage}

\section{Comptes rendus}

\section{(2) OpenEdition}

1 Journals

Édition électronique

URL : http://journals.openedition.org//homme/22345

DOI : 10.4000/lhomme.22345

ISSN : 1953-8103

Éditeur

Éditions de l'EHESS

Édition imprimée

Date de publication : 27 octobre 2009

Pagination : 117-164

ISSN : 0439-4216

\section{Référence électronique}

«Comptes rendus », L'Homme [En ligne], 192 | 2009, mis en ligne le 22 octobre 2011, consulté le 24

septembre 2020. URL : http://journals.openedition.org/lhomme/22345 ; DOI : https://doi.org/10.4000/ Ihomme.22345

\section{(ㄷ École des hautes études en sciences sociales}




\title{
HISTOIRE \& ÉPISTÉMOLOGIE
}

\author{
Françoise Héritier \\ L'Identique et le Différent. Entretiens avec Caroline Broué \\ La Tour d'Aygues, Éd. de l'Aube-France Culture, 2008, I09 p. (« À voix nue »).
}

C

E PETIT LIVRE, coédité par les éditions de l'Aube et France Culture, regroupe une série de cinq entretiens que Françoise Héritier a accordés à la chaîne de radio dans le cadre de l'émission "À voix nue » animée par Caroline Broué.

Françoise Héritier y relate son itinéraire personnel et notamment certains épisodes de l'enfance à l'origine de son goût pour des thèmes qu'elle explorera par la suite. Issue d'une famille qui conservait un fort ancrage rural, elle séjourne longuement au cours de la Seconde Guerre mondiale chez ses grandsparents paternels, en Auvergne. Elle s'initie alors aux tâches de la ferme et à des artisanats d'appoint comme la confection de chapelets (prolongée après-guerre par la décoration des bouteilles de parfum «Opium » d'Yves SaintLaurent!). Elle apprend aussi et surtout à se repérer dans les méandres des rapports de parenté, sujet de conversation favori de ses deux grands-mères, l'une auvergnate, l'autre bourguignonne, tout en prenant conscience des discriminations sexuées très prégnantes à l'époque dans les campagnes françaises. Alors plus tentée par "les ailleurs et les autrefois » que par le monde contemporain, elle se tourne pour ses études supérieures vers l'agrégation d'histoire-géographie, puis c'est la rencontre avec Claude Lévi-Strauss dont elle suit assidûment les séminaires à la $\mathrm{V}^{\mathrm{e}}$ section de l'EPHE. En 1957, l'opportunité d'une mission de longue durée parmi les Panna et les Mossi de l'actuel Burkina Faso la confirme dans sa vocation d'ethnologue. L'Identique et le Différent, titre de l'ouvrage, prête à deux niveaux de lecture. Cette dualité fait tout d'abord référence aux objectifs épistémologiques poursuivis: dénicher des éléments communs à l'espèce humaine en passant les variations culturelles au crible d'une comparaison extensive mais raisonnée. Françoise Héritier réaffirme ici l'ambition anthropologique qui l'anime et qui inscrit son projet intellectuel dans la lignée des Lévi-Strauss, Mauss et Durkheim. La concernant, cette quête vise, comme ses prédécesseurs, à la mise en évidence de lois de portée générale - ses travaux consacrés à l'identité plus forte des germains de même sexe ou à l'inceste du deuxième type en témoignent. Sur un mode plus original, elle s'efforce aussi de dégager, en matière de rapport au réel et de quête de sens, des "invariants". Elle avait déjà abordés ces mécanismes dans Masculin/ Féminin dans l'exploration de ce qu'elle appelle la "valence différentielle des sexes ». Elle les définit au cours de l'entretien (p. 49) comme: «la perception d'un rapport entre concepts qui peut être traité par la pensée de différentes manières ", le rapport servant en l'occurrence de dénominateur commun. Le fait que toutes les 
sociétés se demandent si le corps ou ses organes peut être traité comme une marchandise fait partie de ces invariants, explique-t-elle en guise d'illustration. Ils appellent, selon les contextes culturels, des réponses certes variées mais en nombre fini. Ce que d'aucuns appelleraient des «questions de société" se voient conférées de la sorte une dimension anthropologique, «les ailleurs et les autrefois " apportant des éclairages féconds en vue de la compréhension de "l'ici et maintenant». L'implication de Françoise Héritier ces dernières années dans diverses instances consultatives hexagonales ayant pour thèmes la parité ou le sida trouve ici tout son sens.

À un second niveau de lecture l'identique et le différent sont des catégories princeps au fondement de toute classification binaire structurant la pensée humaine et lui permettant d'appréhender le réel. Françoise Héritier reprend ici de manière plus concise la thèse qu'elle a développée dans des travaux précédents ${ }^{1}$, selon laquelle le rapport entre hommes et femmes est de l'ordre d'une différence primordiale et irréductible qui est apparue comme telle dès l'aube de l'humanité. Cette différence est primordiale car toutes les espèces animales sont divisées selon l'opposition mâle/ femelle et qu'il s'agit là d'un immédiat perceptuel qui s'est imposé aux premiers hommes comme il s'impose aux enfants découvrant le monde ambiant. Ce faisant, l'opposition masculin/féminin, formule archétypale de l'identique opposé au différent, servirait de matrice logique à toutes les autres catégories binaires. Elle est irréductible car elle ne peut être fragmentée plus avant et fait partie en conséquence de ce que Françoise Héritier appelle des "butoirs pour la pensée». Si l'on peut discuter de l'antériorité du binôme masculin/féminin par rapport à d'autres oppositions binaires inscrites dans l'expérience initiale du monde sensible (que l'on pense au binôme chaud/froid), on est malgré tout en présence d'une thèse forte que les chercheurs s'intéressant à la problématique des constructions identitaires devraient sérieusement considérer. Nul mieux que Françoise Héritier n’a prolongé la réflexion structuraliste sur les ressorts de l'identité que Claude Lévi-Strauss et ses élèves avaient esquissée dans les années $1970^{2}$.

Dans le dernier des cinq entretiens, Françoise Héritier revient sur son implication, à partir de la fin des années 1980, dans diverses instances consultatives (Conseil national du sida dont elle assura la présidence, Comité consultatif national d'éthique et Conseil économique et social). Elle exprima dans ces diverses institutions le point de vue de l'anthropologue, s'efforçant de recadrer les projets de lois relatifs notamment au Pacs par rapport aux invariants précédemment évoqués. Certains de ses avis furent critiqués par des tenants de la corporation. Elle eut cependant le grand mérite de montrer qu'à une époque où l'opinion publique enferme de plus en plus la discipline dans "un ailleurs et un autrefois " soi-disant tombés en désuétude du fait de la globalisation, l'anthropologue reste l'un des rares acteurs de la cité à pouvoir exprimer des lois humaines de portée générale, appliquées "partout et toujours".

Pour conclure, ce petit ouvrage, par son énoncé clair et concis, permet au plus grand nombre d'accéder à la pensée féconde d'une grande dame de l'anthropologie française.

\section{Bernard Formoso}

1. Cf. notamment, Masculin/Féminin. La pensée de la différence, Paris, Odile Jacob, 1996 ; et Françoise Héritier, ed., Hommes, femmes, la construction de la différence, Paris, Le Pommier-Cité des sciences et de l'industrie, 2005.

2. Voir le séminaire dirigé par Claude Lévi-Strauss, publié in L'Identité, Paris, Presses universitaires de France, 1977 («Quadrige»). 
C

HuitiÈME livre de Patrick Gaboriau aborde la question des enjeux épistémologiques liés à toute recherche scientifique. C'est un ouvrage qui dérangera le corps des chercheurs complaisants à toute forme de complicité face à l'autorité et au pouvoir en place. C'est un ouvrage salvateur et nécessaire dans une période où les fondements de la recherche sont à penser de manière cruciale.

Pour l'auteur, comprendre la «connaissance comme une activité sociale» (p. 7) revient à décortiquer la recherche dans ses dimensions sociales et politiques, et à s'intéresser d'abord à la question de la question. Pour se faire, il utilise le travail de terrain ethnologique qu'il a effectué auprès des populations sans-logis de Paris, de San Francisco et de Moscou. Pourquoi tel phénomène social fait-il l'objet d'un questionnement et pourquoi tel autre n'en fait-il pas l'objet? La source même de ce questionnement est ainsi analysée au moyen d'un apport théorique solide construit autour de Bourdieu, Foucault, Weber, ainsi que nombres d'auteurs anglo-saxons. En effet, l'auteur s'appuie sur une bibliographie de près de deux cents ouvrages dont un quart en langue anglaise. Les formes de domination et de pouvoir, et leurs rapports au social sont étudiés à partir de la production de la connaissance dans ce qu'il appelle une architectonique des sciences sociales.

Cinquante-cinq points de discussion forment les cinq chapitres de cet ouvrage, ce qui correspond à une construction assez inaccoutumée, bien qu'habituelle chez Patrick Gaboriau $^{1}$; et chaque point fait l'objet d'une réflexion ou d'une discussion, ce qui facilite une lecture partielle. La progression de l'ouvrage nous conduit d'une considération générale sur l'épistémologie à une mise en forme pragmatique du rapport entre journalisme et sciences sociales, pour aboutir à l'appel d'une vigilance épistémologique.

Dans un premier temps, Patrick Gaboriau aborde les questions de la «neutralité scientifique", celles des vertus du chercheur face au pouvoir politique, celles du rapport étroit entre politique et recherche, celles de la construction de la connaissance, supposant une réflexion sur la construction des moyens et des outils de cette connaissance et offrant, dès lors, une forme de "connaissance de la connaissance". Pour cela, l'auteur évacue un certain nombre d'a priori, à commencer par la neutralité du chercheur, la cohésion du "monde scientifique", et montre en quoi les médias sont eux-mêmes pris dans le rapport au politique: "dans les médias on ne compte plus les phrases qui débutent par "comme disent les sociologues..." " (p. 31).

Il poursuit son argumentation en prenant comme exemple une étude statistique sur le dénombrement des personnes sans logis en France. L'organe officiel, l'INSEE, serait «l'objet d'enjeux et de lutte pour éluder les débats sur [l'] objectivation [des statistiques]» (p. 35). Sans dénigrer l'outil - «une formation en statistique paraît indispensable pour l'ethnologue ou le sociologue» (p. 37) -, l'auteur démontre une « logique sociale à l'œuvre au service d'un pouvoir» (p. 36). L'absence d'un questionnement préalable sur la notion même de $\mathrm{SDF}$, qui ne trouve finalement sa logique que dans l'ordonnancement du monde par les chiffres, et l'absence d'une comparaison

1. Voir notamment Patrick Gaboriau \& Daniel Terrolle, SDF. Critique du prêt-à-penser (Toulouse, Privat, 2007), qui a reçu le prix 2008 Bigot de Morogues décerné par l'Académie des Sciences morales et politiques. 
internationale (etc.) posent à l'évidence le problème du rapport entre pouvoir et sciences sociales. "La définition même du "sans-logis" est un enjeu social » (p. 44), écrit-il, avant d'ajouter d'une façon plus générale que la "tâche essentielle n'est pas de penser les catégories constituées, mais de rechercher comment certaines catégories sont devenues dominantes, et de s'attarder sur les moments de passages et de ruptures, qui renseignent sur les transformations sociales et mentales d'une société" (p. 46). L'utilisation de l'objet « sans abris » est donc particulièrement éclairante pour illustrer le travail de déconstruction conceptuelle que doit faire tout chercheur lorsqu' il aborde un objet quel qu'il soit.

Dans le chapitre suivant, Patrick Gaboriau poursuit sur la construction de la connaissance scientifique à partir des lectures de Max Weber, qui ont inspiré le titre de cet ouvrage. Sommes-nous simplement «tributaire[s] de la pensée de notre époque (p. 61) ? Si c'est le cas, l'acte critique de l'activité scientifique s'inscrit-il dans une démarche d'objectivation elle-même sous-tendue par un cadre rigide et non franchissable? "Le chercheur n'est pas extérieur au monde mais dans celui-ci, soumis dès lors aux règles qu'il découvre" (p. 66). Il est temps de reconsidérer Weber à la lumière de l'évolution de la pensée et de tenir pour acquise l'idée selon laquelle « la politique est un constituant, c'est-à-dire un élément déjà là si l'on considère la dynamique de la découverte intellectuelle, à savoir le processus même du travail de recherche" (p. 71). En effet, la « sociologie weberienne est l'expression politique d'une forme de sociologie qui met à distance les observations de terrain et s'intéresse en bureau aux fonctionnements institutionnels» (p. 79). Patrick Gaboriau est en cela fidèle à ses préoccupations de terrain, et l'auteur de considérer à plusieurs reprises que le terrain est une condition sine qua non de la recherche en sciences sociales. De même, la responsabilité du chercheur dans sa société n'est pas "d'endosser le rôle de valet des autorités»(p. 82). Pourtant, les crédits de recherche ne font-ils pas apparaître une pointe d'opportunisme et de complaisances? Car, lorsqu'il écrit que «le choix même de l'objet de recherche est un acte politiquement situable» (p. 94), l'auteur énonce les conditions d'une forme d'engagement sui generis: les "outils de vigilance épistémologique (l'observation directe, les études de terrain, l'enquête, la réflexion réflexive...) ne suffisent pas; c'est aussi l'engagement personnel du chercheur qui importe" (p. 99). De ce point de vue, ce livre transcende le guide méthodologique, pour aborder les fondements de la recherche de terrain au-delà des seuls modes de collectes de données. On en vient à se demander si les ouvrages de "méthode" ne seraient pas inconsciemment écrits pour asseoir l'acte de domination du pouvoir en place et de la reproduction sociale universitaire et scientifique?

Le quatrième chapitre porte sur la relation entre journalisme et sciences sociales. Pour l'auteur, il s'agit, en comparant trait pour trait son propre travail de terrain avec celui d'un journaliste, d'écarter toute ambiguïté face à deux conceptions clairement opposées du rapport à la réalité. En effet, les tenants et les aboutissants des métiers de journaliste et de chercheur s'opposent en cela que la méthode d'approche du premier découle d'intérêts immédiats en termes de représentation, de carrière, de vente, de popularité, alors que le chercheur sera animé par une volonté heuristique; le temps d'enquête est par exemple très différent dans chacun des deux cas; le rapport à l'éthique est autrement plus sérieux chez le chercheur. Mais il y a plus grave, lorsque des sociologues amalgament le travail de l'ethnologue à celui du journaliste. Nous voyons que des apories intellectuelles sont parfois présentes chez les penseurs officiels. L'ethnologue se doit de remettre en cause les assertions du journaliste, de tordre le cou aux préjugés hissés au rang de "vérités " qui amorcent une caricature sociologique envers un groupe qui n'en est pas un. L'auteur analyse une construction sociale à l'œuvre en pointant ses implications 
sociales et politiques. Davantage écoutés, les propos du journaliste offrent un cadre de pensée censé éclairer le "grand public" tout en participant à la construction d'un imaginaire collectif.

Certes, tous les journalistes ne sont pas à mettre dans le même panier. (Cela est aussi valable pour les sociologues.) Pensons à John Howard Griffin, à Günter Wallraff ou à Jack London, et à leur travail d'investigation pour ne pas douter de leur sincérité. Par ailleurs, quel ethnologue ou sociologue ne serait pas tenté par le pouvoir si jamais on lui en faisait la promesse ? Cela n'est pas contredit par Patrick Gaboriau qui, en acceptant la coexistence de plusieurs vérités, oriente son dernier chapitre sur la nécessité d'un esprit d'ouverture. La connaissance a atteint ses limites alors même qu'elle dépend pour une large part de l'état des connaissances et de l'état d'esprit d'une époque. Pris dans l'air du temps, nous ne pouvons percevoir un niveau supérieur de connaissance car nous sommes prisonniers d'un hic et nunc socialement construit. Aussi l'auteur de se questionner sur ce dépassement en se demandant : "le domaine de connaissance institué par une épistémé particulière peut-il dépasser et contredire l'épistémé qui le construit?» (p. 143). Ce dépassement n'est possible que si les conditions du questionnement sont elles-mêmes questionnées. Cela nécessite d'esquiver les instances de contrôle et de réglementation qui "autorisent, canalisent et limitent les possibilités de production et de diffusion» (p. 146).

L'auteur est animé par le credo de l'engagement pour lequel " un combat politique essentiel en sciences sociales consiste à lutter pour imposer des formes d'objectivation du monde social» (p. 149). En cela, il souligne son désaccord avec les formes de verrouillage de la pensée qu'il qualifie de "véritable service d'ordre du savoir" (p. 151). De l'évaluation à l'attribution de crédit, de la distribution de satisfecit à l'autocensure, les formes de contrôle social sont multiples. Patrick Gaboriau propose de sortir de ce carcan par une vigilance épistémologique. Celle-ci doit commencer par une reformulation des questions, une attention face à la collusion et un évitement de toute complaisance pour atteindre ce qu'il nomme une architectonique de la critique.

Noël Jouenne 
M son ouvrage Shamanism, Colonialism, and the Wild Man ${ }^{1}$ qui traite de l'impact de la colonisation sur le shamanisme et la médecine populaire en Colombie. Dans Walter Benjamin's Grave, il poursuit l'exploration de nouvelles façons d'écrire une œuvre anthropologique, qu'il avait notamment expérimentée dans son précédent ouvrage My Cocaine Museum ${ }^{2}$. Il s'agit dans ce nouveau recueil d'articles, publiés dans les dix dernières années, de trouver une écriture déceptive qui rende le monde social étrange, même lorsqu'il est bien connu, plutôt que de proposer des explications ou des analyses, en substituant ainsi au modèle de l'autorité scientifique celui de la construction littéraire. Les chapitres de cet ouvrage sont autant de paysages moraux, des aller et retour entre des références théoriques principalement européennes de la fin du XIX ${ }^{e}$ siècle (Freud-Marx-Nietszche) et du début du XX (Bataille-Benjamin), et des vignettes issues des terrains exotiques fréquentés par l'auteur ou par d'autres ethnologues, à l'intention d'un public nordaméricain. Manifestement, le ton et le montage de ces textes importent autant à l'auteur que les propositions théoriques qu'ils contiennent et l'unité de l'ensemble ne tient qu'au retour régulier du propos sur les questions de la narration, de l'écriture et de l'autorité.

Les quatre premiers chapitres de ce recueil baroque sont ainsi constitués, pourrait-on dire, du Tombeau de Walter Benjamin, de l'Anthropologue Inconnu, de celui de Georges Bataille et enfin d'un autoportrait au bord de la mer (un Tombeau de l'enfance, en quelque sorte). La figure de Walter Benjamin est abordée avec la visite de Taussig au cimetière de Port-Bou qui lui permet à la fois de rencontrer les passeurs avec qui Walter Benjamin avait franchi les Pyrénées et de réfléchir, sur la tombe de celui-ci, à son suicide. C'est également pour lui l'occasion de revenir sur la théorie de la narration proposée par Benjamin dans son essai sur Leskov. Michael Taussig est un bon connaisseur des textes de Benjamin, qu'il a notamment utilisés dans son article « Terror as Usual: Walter Benjamin's Theory of History as a State of Siege » ${ }^{3}$. Or ce chapitre d'ouverture est avant tout une flânerie à la tonalité d'un romantisme sombre. Arrivé au terme de l'article, on s'étonne de la minceur conceptuelle de la référence à Benjamin qui donne pourtant son titre à l'ouvrage.

Bien plus stimulant est le deuxième texte, dans lequel Taussig réfléchit aux notes prises par un enquêteur inconnu au cours de ses entretiens avec un vieil Indien de Colombie, Tomás Zapata. Que se passe-t-il quand l'anthropologue disparait, et qu'il ne reste de son travail qu'un paquet de notes et d'enregistrements sonores? C'est, pour l'auteur, l'occasion de rappeler que sur le terrain, c'est toujours par le biais d'histoires (parfois appelées "cas») que l'information est transmise à l'enquêteur, dont le travail consiste ensuite à faire médiation entre ce savoir privé et un public cultivé. L'anthropologue, en nommant "observations scientifiques" ces récits, est trop souvent aveugle au fait qu'il raconte les histoires et transmet les savoirs d'autres gens (eux-mêmes qualifiés d'informateurs). La narration en situation d'enquête apparaît ainsi comme le lieu de création conjointe d'un espace de transfert interculturel, entre poésie, mémoire et langage, entre passé et présent.

1. Chicago, University of Chicago Press, 1987.

2. Chicago, University of Chicago Press, 2004.

3. Social Text, 1989, 23:3-20. 
La référence à Georges Bataille et à sa reprise de l'analyse maussienne du don dans La Part maudite (chap. III) sont assez déroutantes pour les lecteurs qui connaissent bien l'ouvrage. La présentation par Michael Taussig de la position de Bataille sur l'échange, la part du diable et de ses différences avec Marcel Mauss et Claude Lévi-Strauss est tout à fait convaincante, mais déjà bien connue en France ${ }^{4}$.

Le quatrième chapitre est tout entier empreint d'une nostalgie post-benjaminienne, les souvenirs d'enfance de l'auteur au bord de la mer lui permettant à la fois de saisir les changements sociaux à Sydney et de proposer une certaine phénoménologie de l'être-au-monde. Les derniers chapitres de l'ouvrage se focalisent sur des objets plus habituels dans les travaux de Michael Taussig, et notamment le shamanisme et la sorcellerie. Pour lui, les tours de passe-passe des sorciers ne sont pas plus sidérants que ceux qu'institue l'écriture des anthropologues qui en rendent compte. Si le projet même de l'ouvrage justifie la liberté du parcours conceptuel suivi, loin de toute érudition monographique, le lecteur s'étonne parfois de raccourcis approximatifs - par exemple lorsque Taussig suggère qu'il faudrait ouvrir à l'analyse la relation de Franz Boas avec son informateur George Hunt (p. 231, note 29), alors même qu'il existe précisément des travaux à ce sujet ${ }^{5}$. En définitive, la substitution de l'évocation (empirique ou théorique) à l'administration de la preuve laisse dans cet ouvrage un goût d'inachèvement qui, s'il est probablement désiré par l'auteur, fait craindre que les textes de ce recueil apportent à leurs lecteurs une moindre stimulation heuristique que les précédents livres de Michael Taussig.

Michel Naepels

4. Cf. par exemple Dominique Lecoq \& Jean-Luc Lory, eds, Écrits d'ailleurs. Georges Bataille et les ethnologues, Paris, Éd. de la Maison des sciences de l'homme, 1987.

5. Voir notamment Judith Berman, " "The Culture as it Appears to the Indian Himself" : Boas, George Hunt and the Methods of Ethnography ", in George Stocking, ed., Volksgeist as Method and Ethic, Madison, University of Wisconsin Press, 1996 : 215-256, et Ira Jacknis, "George Hunt, Collector of Indian Specimens", in Aldona Jonaitis, ed., Chiefly Feasts, New York, American Museum of National History / Seattle, University of Washington Press, 1991 : 177-224. 
C

ET OUVRAGE COLLECTIF dirigé par Tiphaine Barthélemy et Maria Couroucli est issu d'un colloque organisé en 2005 par la section d'Anthropologie sociale du CTHS. Quinze auteurs, principalement des ethnologues, des historiens, des sociologues et des spécialistes de la littérature, contribuent à alimenter une réflexion sur la question de l'écriture ethnographique et de ce qui la distingue et/ou la rapproche de l'écriture de voyage.

Ces deux formes de mise en récit de la rencontre avec l'Autre et avec l'Ailleurs sont posées en miroir, pour faire apparaître des différences et des similitudes entre elles. Nous découvrons, au fil de l'ouvrage, que les frontières qui reposent sur les dualismes objectivité/subjectivité, science/littérature sont poreuses et souvent inaptes à rendre compte des multiples passerelles qui les lient et des différences plus subtiles qui les distinguent. Que cache l'aversion des ethnographes vis-à-vis des voyageurs? Sans doute une fascination/répulsion pour ce qui est trop proche et en même temps tenu pour distinct.

Les oppositions entre "rationalité et sensibilité, savoir et intuition, recherche méthodique et vagabondage " se mêlent chez tous les voyageurs, qu'ils soient ou non ethnographes, annoncent Tiphaine Barthélémy et Maria Couroucli, dans leur propos introductif (p. 14). Cet ouvrage prend donc acte de la déconstruction de l'objectivité de l'ethnologie, en ce qu'elle traduit la réalité empirique et propose une construction rhétorique orchestrée par l'ethnographe. Cependant, les auteurs prennent soin de ne pas réduire le texte ethnographique à une figure de style ou à une fiction, à la manière de James Clifford et de Clifford Geertz. De même, ils ne dénient pas au récit de voyage ses qualités empiriques. L'ouvrage propose d'aller plus loin en tâchant de restituer la complexité de l'observation ethnographique et de sa mise en texte, en posant la question des liens entre le fond et la forme, entre les procédés d'écriture et les faits exposés.

La réflexion se nourrit essentiellement de l'analyse de textes ethnographiques écrits dans un style littéraire et de récits de voyage. Sont tour à tour évoquées des figures d'aventuriers, d'explorateurs savants, de missionnaires, d'écrivains voyageurs et d'ethnologues. Les articles soulèvent la question du poids des règles stylistiques et des conventions académiques dans le processus de reconnaissance d'un texte ethnographique, de même que celle de la légitimité de l'auteur et de l'autorité de son discours. Ils interrogent également et surtout les thèmes centraux du voyage, du dépaysement et des "connaissances qui sous-tendent les regards portés sur l'Autre» (p.13).

Une première partie, "L'anthropologie contemporaine: entre argumentation et narration ", regroupe des articles relativement disparates, et aurait sans doute mérité une introduction pour en éclairer la cohérence. Quelques axes de réflexion s'en dégagent néanmoins, comme le rapport ambigu que les ethnologues entretiennent avec le voyage ou bien avec les modes de narration littéraire. S'intéressant à trois textes "hybrides» écrits par des ethnologues, à la première personne, (L'Afrique fantôme de Michel Leiris, Tristes Tropiques de Claude Lévi-Strauss et Blackberry Winter. My Earlier Years de Margaret Mead), Gérard Toffin revisite l'aversion affichée des ethnologues pour le voyage et en propose une lecture plus nuancée. Dans ces livres qui possèdent une forme narrative très différente de l'écriture ethnographique 
académique, chaque auteur se positionne, à sa manière, en rupture avec les marchands d'exotisme, tout en dotant le voyage d'une vertu littéraire, introspective et scientifique. À sa manière également, Geneviève Bédoucha réhabilite le mode narratif « littéraire ". À partir de l'analyse comparée de deux ouvrages portant sur les communautés villageoises de l'Euphrate, l'un écrit par un doctorant en anthropologie (Shâkir Mustafa Salïm) et l'autre par un voyageur aventurier (Wilfred Thesinger, Les Arabes des marais), l'auteure montre la complémentarité de ces textes, au demeurant fort différents. Chacun témoigne d'une observation fine de la réalité sociale que le texte ethnologique peut réconcilier, en alliant la rigueur conceptuelle et un mode narratif qui restitue l'épaisseur du sensible.

"Ethnologues et voyageurs évoluent dans les mêmes parages" (Toffin, p. 34) et les textes d'ethnologues témoignent parfois de cette proximité dans l'écriture. Cependant, les auteurs rappellent aussi que le terrain ethnographique ne saurait se confondre avec le voyage. La contribution de Michèle Baussant sur le cheminement de l'écriture ethnologique, ou bien, dans un tout autre registre, celui de Sergio Dalla Bernardina sur les procédés discursifs de la littérature missionnaire, montrent qu’ " Entre le regard et l'écriture s'interposent des filtres multiples, parmi lesquels les lectures, l'idéologie, le statut de l'auteur, jouent un rôle de premier plan» (p. 16).

Dans une deuxième partie intitulée «Lectures anthropologiques et récits de voyage ", le récit de voyage devient objet anthropologique. Les écrits analysés sont le fait d'écrivains comme Alphonse de Lamartine et Gérard de Nerval, Ella Maillart, Laurence Durrel et Henry Miller, ou bien de scientifiques, tels Charles Varat ou encore Charles de la Condamine. À la lumière de leur description, les pays arpentés apparaissent comme de "grands espaces d'investissement de l'imaginaire occidental» (Bonin, p. 148). En restituant l'ailleurs, les voyageurs disent aussi quelque chose d'eux-mêmes et de leur appartenance à une époque, à un pays, à un milieu social, vis-à-vis desquels ils se positionnent en même temps qu'ils en témoignent. Charles Varat, missionné par le gouvernement français pour une collecte ethnographique en Corée, apparaît sous la plume de Guillaume Bonin comme un émissaire de la Troisième République, pétri de progrès et de scientisme. Selon Adriana Cabrera Müller-Wirth, Charles de la Condamine (1701-1774), également marqué par son siècle, livre dans ses écrits des indications à la fois sur l'état de la science et sur la perception mythifiée des réalités péruviennes, à l'heure de la conquête du Nouveau Monde. Pour Yann Bouvrat, les écrits de Lamartine et Nerval sur les Maronites et les Druzes au Liban permettent de comprendre l'état de l'opinion française face à la question de l'Orient au milieu du XIX ${ }^{e}$ siècle.

Le contexte historique et le parcours singulier des auteurs influencent les écrits en même temps qu'ils les éclairent. Cette lecture historiographique des récits de voyage permet également d'interroger les savoirs produits sur les réalités sociales observées et de réintroduire la question de la distinction entre ethnographes et voyageurs. L'ouvrage met en évidence que ces derniers sont animés par une volonté sincère d'interroger l'altérité et tentent toujours de se départir, avec plus ou moins de succès, d'un certain nombre d'idées préconçues. C'est le cas d'Ella Maillart, écrit Tiphaine Barthélémy, qui défend un principe méthodologique assez proche de celui préconisé par Bronislaw Malinowski pour «le travail en plein air» (p. 170). Il apparaît également que les voyageurs élaborent leur savoir dans l'expérience de la rencontre, sans oublier de le confronter avec les écrits existants, ce qui peut donner lieu à des observations assez fines ou bien à des analyses bien étayées. Cependant, certaines différences significatives demeurent, qui tendent à relativiser la «subjectivité » de l'ethnographe. La plus importante tient au fait que les voyageurs font souvent preuve d'un "fervent ethnocentrisme à l'envers " (Barthélémy, p. 173), mêlant désir d'évasion et tendance 
à l'idéalisation, avec comme motif ultime et premier de l'écriture, la quête de soi. Les descriptions d'Ella Maillart (Parmi la jeunesse russe), si fines soient-elles, se greffent sur une trame narrative constituée par les impressions de l'écrivaine. Le voyageur est mobile, l'ethnographe est sédentaire et doté d'un outillage conceptuel et méthodologique.

Cependant, les voyageurs élaborent des savoirs et participent, à leur manière, à la définition des altérités. Comme le note Maria Couroucli, à propos de la Grèce, les thèmes dont ethnographes et voyageurs se saisissent sont parfois étrangement proches (hospitalité, religion populaire, famille, rôles masculins et féminins) (p. 179). Les écrivains semblent élaborer et même adhérer à des mythes identitaires que les ethnologues s'attachent en retour à étudier, tout en prenant part au débat sur l'identité. Les frontières ne sont pas étanches et il faut bien reconnaître que les ethnologues mobilisent parfois les écrivains comme source de connaissance. Aussi arrive-t-il parfois que l'ethnologue "rame à contre-courant " du parti pris des voyageurs amoureux, "tout en cultivant le même champ" (p. 185). Il arrive également que les ethnologues se saisissent des récits de voyage comme «stimulant mémoriel » et source d'inspiration, pour ensuite les déconstruire, voire les contredire : tel est le cas développé par JeanPierre Jardel, à propos de l'utilisation faite par Claude Lévi-Strauss d'un texte de JeanBaptiste Wilkinson (peintre-voyageur) sur la Martinique.

La question des passerelles, entre objectivité et subjectivité, entre science et art, entre rigueur scientifique et imagination littéraire (Jardel, p. 194), traverse donc l'ensemble de l'ouvrage. Elle est abordée, dans la troisième partie, sous l'angle particulier de la rhétorique des écrits, des procédés discursifs et narratifs, des traditions et conventions textuelles. Difficile ici de dégager des lignes de force significatives. Il en ressort néanmoins que la mise en mot du réel n'est jamais uniforme et recèle une pluralité de modes narratifs et de logiques de représentations, que s'empruntent mutuellement ethnographes et voyageurs. L'écriture documentaire de Nicolas Bouvier sur le Japon est un véritable " précis de perception et d'interprétation des différences culturelles", qui articule étroitement «diachronie et synchronie, faits objectifs et impressions subjectives, narration et description" (Aki Taguchi, pp. 269 et 278). Les intellectuels arabes à Paris vont à la rencontre de la description ethnographique pour saisir le familier et le quotidien dans des dimensions jusque-là ignorées (Leonardt Santini, p. 264). De même, l'épaisseur du vécu et de la singularité de l'ethnologue transparait dans les écrits, parfois même sous l'homogénéité de surface de l'écriture académique (Jean-Pierre Martinon, p. 216).

Dans ce tour d'horizon hétéroclite et parfois redondant, les ethnologues et écrivains se trouvent ainsi confrontés en miroir à leur propre pratique d'observation et d'écriture. Ce retour sur soi, réflexif et utile, comporte néanmoins des limites, sans doute liées à la difficulté d'appréhender des réalités textuelles dans une perspective anthropologique. Il manque en quelque sorte ici la mobilisation d'un outillage conceptuel et méthodologique, tel que développé par Daniel Fabre et d'autres, pour faire parler les écritures. S'intéresser à la reconfiguration du réel et au rapport à la vérité aurait peut-être permis d'aborder les différences entre écritures fictionnelles et ethnographiques de manière plus convaincante. En outre, on aurait aimé que soient mieux restituées les réalités sociales observées, en tant qu'objet de savoirs et de représentations plurielles, pour saisir l'articulation entre le texte et le monde de référence, qui n'est pas de même nature dans la littérature et dans les sciences sociales. Cependant, la thématique abordée dans cet ouvrage est intéressante et offre des pistes utiles, qui invitent à poursuivre la réflexion. 
$\mathrm{F}$ ONDÉ sur une recherche qui a débuté voilà quarante ans, l'ouvrage que nous offre Marion Segaud témoigne d'une persévérance exemplaire. Par son appareil critique, les réflexions développées, Anthropologie de l'espace se présente comme un véritable instrument de travail pour une discipline à la croisée des sciences sociales. S'il est souvent question d'anthropologie urbaine, les autres disciplines comme la psychologie, la sociologie, la géographie ou la philosophie n'en sont pas exclues pour autant. Car, cette jeune discipline qu'est l'anthropologie de l'espace puise ses ressources dans le vaste champ des sciences humaines. Pour l'auteure, c'est « un outil de veille pour des spatialités mondialisées" (p. 13).

Les deux premiers chapitres retracent l'histoire et le contexte du développement de l'anthropologie de l'espace en France et en cela sont passionnants. L'auteure reprend le récit des écoles d'architecture à l'orée des années 1970. À cette époque, quelques architectes interrogent les sciences sociales à propos, notamment, du rapport entre la forme, l'espace, l'individu et l'environnement. Les travaux précurseurs, outreAtlantique, sont sollicités afin de mieux percevoir les différences avec les recherches françaises : "Si le contexte lié à l'environnement construit est relativement semblable, le contexte disciplinaire, lui, est différent. La production architecturale du logement de masse, sous la pression de l'industrialisation du bâtiment et du développement de l'urbanisation, engendre un certain mécontentement" (p. 20). À la fin des années 1960, un certain nombre d'études vont permettre d'éclairer la vie quotidienne des ouvriers (Paul-Henry Chombard de Lauwe), des populations en bidonville (Colette Pétonnet), des nouveaux résidents en pavillon (Nicole Haumont, Henri Raymond). Il en sera de même du côté de la psychologie des modes d'appropriation (Abraham Moles, Perla KorosecSerfaty, Claude Leroy, Monique Eleb). Sans unité d'école, l'anthropologie de l'espace se constitue peu à peu.

Les références abondent et ne sont pas neutres. Elles éclairent davantage sur le parcours de l'auteure, et en cela, construisent une histoire de la discipline singulière. Par exemple, lorsque Marion Segaud aborde la question sous l'angle de l'école de Chicago, ou du Dictionnaire de l'ethnologie et de l'anthropologie de Pierre Bonte et Michel Izard, on s'étonne de ne pas trouver l'article de Patrick Williams sur l'anthropologie urbaine. Si cette discipline, comme le prétend l'auteure, est née à Chicago, il faut reconnaittre que sa cousine française ne lui doit rien, tout du moins dans les premiers temps ${ }^{1}$. Ici, les principales références utilisées proviennent de textes dont les auteurs - avec lesquels Marion Segaud a parfois collaboré - officient dans des écoles d'architecture. Cela témoigne d'une fidélité sur le long terme mais oriente inévitablement son discours. D'ailleurs, l'auteure ne s'en cache pas puisqu'elle écrit que "ce panorama rapide est bien sûr de parti pris " (p. 40). Le débat se prolonge tout au long du deuxième chapitre à propos du rapport entre espace et politique, toujours en lien avec l'architecture. Concernant les espaces publics, "espaces où se forme l'opinion publique, base de la démocratie " (p. 44),

1. Cf. Jacques Gutwirth, "L'anthropologie urbaine en France", inédit, 2007. Cet article numérique propose une interprétation beaucoup plus vaste sur l'histoire de l'anthropologie urbaine.

[http://halshs.archives-ouvertes.fr/halshs-0026 7536/fr/]. 
l'auteure convoque Jürgen Habermas puis Claude Lévi-Strauss, Walter Benjamin, dans laquelle ceux qui pensent l'espace (qu'ils soient chercheurs ou constructeurs) pourront venir puiser» (p. 48). Car «l'espace est un enjeu forcément stratégique pour les groupes ou les individus" (p. 50).

Ces outils font l'objet des quatre chapitres suivants. Ils sont organisés autour "d'universaux " déclinés dans cet ordre: " habiter ", " fonder ", " distribuer et classer " et, "transformer et reformuler». Chaque concept est défini, discuté, argumenté et exemplifié selon un rythme variable: la notion d'habiter est traitée, par exemple, en trente-deux pages. L'auteure commence par définir cette notion grâce à l'apport de Heidegger, puis de Bachelard: "Habiter c'est, dans un espace et un temps donnés, tracer un rapport au territoire en lui attribuant des qualités qui permettent à chacun de s'y identifier» (p. 65). On entre ici dans les fondements de l'humanité, par des «détours» (psychologie, phénoménologie, sociologie, philosophie), qui donnent à voir une anthropologie dans sa dimension multiculturelle. Ainsi, les différentes formes d'habiter sont passées en revue, de la double résidence à l'habiter nulle part, des pratiques d'habiter en France, au Japon ou en pays mongol. Chaque illustration est soulignée de quelques références : libre au lecteur d'y apporter sa pierre.

Le concept "fonder" est développé à partir d'une réflexion philosophique, car ses fonctions sacrées et symboliques sont d'importance. De l'Égypte au Proche-Orient ancien, en passant par Rome puis le Vietnam, Marion Segaud fait appel aux archéologues pour mesurer la part du symbolique dans les récits de fondation. L'auteure dresse le parcours de la délimitation jusqu'au seuil, car "délimiter c'est donner du sens à l'étendue et cela n'a pu se faire qu'une fois que l'homme a su manier la dimension symbolique»(p. 119). Cet invariant culturel qu'est le seuil sert à organiser les relations sociales. Plus qu'un travail théorique, l'ouvrage est également

un guide pour l'architecte. Ça et là, des remarques soulèvent une approche engagée $\mathrm{du}$ rapport à l'habiter. Lorsque, par exemple, Marion Segaud écrit : «Identifier l'entrée de son logement est une nécessité psychologique pour l'habitant» (p. 124), elle ne peut s'empêcher d'ajouter que " c'est d'ailleurs ce qu'ont bien compris les rénovateurs des cités de logements sociaux, en marquant les halls d'entrée de signes architecturaux distinctifs" (id.). S'agit-il d'un constat ou d'un appel ? Comme précédemment, les exemples sont parfois étayés par un schéma ou un plan empruntés à différents auteurs.

Émile Durkheim et Marcel Mauss occupent une place de choix dans le chapitre consacré aux notions "distribuer" et " classer». Pour eux, l'esprit de classer est un acte culturel. Une discussion s'ensuit sur la dimension dualiste et figée de telles notions, aujourd'hui remises en cause. Pour Philippe Descola, "ces positions n'ont pas l'universalité qu'on leur a attribuée depuis la naissance de l'anthropologie. Il s'agit en effet, selon lui, d'une construction épistémologique de catégories, issues d'une tradition savante, née dans la pensée moderne occidentale, et sans signification pour ceux qui ne partagent pas cette forme de pensée " (p. 131). Ces réflexions nous conduiront à l'échelle de l'espace domestique. Sont abordées la distribution des pièces et des personnes, selon le sexe, la parenté et le statut social. S'agissant de l'espace privé, l'auteure signale l'importance de la prise en compte de ces éléments dans la conception architecturale, et donne quelques conseils aux concepteurs. Ce chapitre aborde également la question de la ségrégation, terme "très idéologisé et instrumentalisé » (p. 145). À notre étonnement, l'article fondateur de Jean-Claude Chamboredon et Madeleine Lemaire ${ }^{2}$ n'y figure pas.

2. Cf. "Proximité spatiale et distance sociale. Les grands ensembles et leur peuplement ", Revue française de sociologie, 1970, 1:3-33. 
"Transformer » et " reformuler» sont les deux derniers concepts universaux que l'auteure aborde dans un chapitre assez dense constitué d'écrits, centrés sur la notion de reformulation. La dimension historique est centrale car "l'analyse de l'interface entre dispositifs et pratiques s'inscrit dans un monde mouvant où les formes spatiales comme les formes sociales s'ajustent en permanence dans la vie quotidienne» (p. 162). Marion Ségaud propose une méthode d'analyse suivant trois niveaux : celui de l'innovation pilotée par le ministère de l'Équipement, celui de la réception du bâti par les habitants, enfin celui d'une anthropologie architecturale fondée sur l'étude historique des permanences de formes architecturales. Chaque sous-partie est largement documentée par des exemples puisés dans la littérature ethnologique, toujours illustrée de croquis, de dessins et de plans. Le thème de l'acculturation est traité en filigranes et revient dans le rapport des formes aux migrants. L'auteure aborde également la question de la "reformulation exogène violente" dans le cas des populations déplacées sous la contrainte. En prenant l'exemple de
l'Algérie sous l'emprise de la colonisation française, elle montre l'importance du rapport au sol et des conséquences des déterritorialisations. Une anthropologie de l'espace requiert un sens critique face à la relation entre l'ordre social et l'ordre spatial. La fin du chapitre ouvre sur une réflexion politique que l'on pourrait formuler à partir de la notion de partage, davantage destinée au praticien, mais qui ne peut qu'éclairer le chercheur. Car de partage il est question, entre le bâtisseur et l'habitant, le promoteur et le planificateur, sur fond de transformations sociales permanentes.

Enfin, les chapitres sont illustrés de dessins dont nous n'avons personnellement pas perçu l'intérêt. Une bibliographie conséquente (294 références) témoigne de l'érudition de l'auteure en en pointant les choix théoriques ainsi que les amitiés longues et fidèles. Un index thématique et un index des lieux et des populations accordent à cet ouvrage une place de choix aux côtés des utilitaires et ouvrages de référence.

Noël Jouenne 
Christophe Le Digol, ed.

Dictionnaire de sociologie

Préface de Howard S. Becker

Paris, Albin Michel-Encyclopædia Universalis, 2007, 920 p., index.

O N PEUT ATTENDRE d'un dictionnaire de sociologie ce que l'on attend habituellement d'un répertoire commenté d'auteurs, de concepts, de références ou de thèmes savants: le compte rendu synthétique et relativement complet d'une production disciplinaire; une définition contextualisée des problématiques intellectuelles qu'elle recouvre. Si l'on peut en juger à partir d'une lecture sélective, ce substantiel volume répond largement à ces réquisits. Il rassemble à cette fin les contributions les plus significatives d'une première édition, datant d'une dizaine d'années, et des contributions inédites de spécialistes portant sur des concepts consacrés ou émergents, sur des travaux et des auteurs reconnus ou en passe de l'être, sur des héritages intellectuels débattus ou en discussion. La préface d'Howard Becker rappelle à juste titre la gageure de ce type d'entreprise et la proposition paradoxale qu'elle doit mettre en forme: penser l'hétérogénéité d'une discipline indisciplinée, soumise à des concurrences variables dans leurs formes et dans leurs contenus, et, parallèlement, rendre compte des consensus disciplinaires sans lesquels critiques et controverses sont inconcevables. Deux ambitions évidentes justifient, pour Becker, cette publication. La volonté de rendre raison de ces différences et de ces tensions à travers l'histoire dont elles sont le résultat, et l'intention d'éclairer à nouveaux frais cette réalité parfois négligée que les sociologues, dans leur diversité, n’ont jamais cessé de travailler sur des groupes et des phénomènes collectifs. Des tendances de fond et des reconfigurations sont, dans cette logique, rappelées par une grande partie des contributions. Cellesci mettent en évidence les continuités et les discontinuités du travail sociologique qui ont souvent un rapport avec la constitution de traditions d'analyse nationales et les appropriations différenciées de ces traditions. Quels que soient les terrains et les thèmes traités par les différents auteurs, plusieurs évolutions générales sont signalées : la dévaluation constante en sociologie de représentations abstraites, téléologiques, généralisantes issues d'autres idiomes savants (la biologie, le droit, les «sciences morales et politiques ") ; la consolidation d'un répertoire d'analyse historicisant focalisé sur la genèse et la transformation des groupes et des espaces sociaux ; l'investissement collectif des instruments de l'analyse réflexive, notamment sous la forme d'une sociologie de la connaissance sociologique et d'une histoire sociale des disciplines académiques.

Il n'est pas surprenant de retrouver au sein des notices une description des innovations et des débats qui ont fait date et qui marquent rétrospectivement un état passé, voire dépassé, de la connaissance sociologique. Un grand nombre de représentations interchangeables du monde social, qui ont pu emprunter leurs mots ou leurs méthodes à des spécialités socialement plus légitimes (le droit ou la médecine par exemple), apparaissent ainsi, après une phase d'autonomisation des instruments de l'analyse sociale, comme des catégories présociologiques. La trajectoire historique de certains concepts résume ce mouvement de socialisation scientifique du vocabulaire sociologique. Les métaphores de l'organisme et les analogies physiologiques sur lesquelles se sont fondées des rationalisations stabilisatrices de l'État (Edmund Burke, Joseph de Maistre, Louis de Bonald) font partie de ces schèmes d'analyse partagés progressivement, ressaisis comme des schèmes spontanés à la 
faveur d'un travail polémique sur la langue et ses impensés ("Organicisme», Pierre Birnbaum) ${ }^{1}$. L'abolition de ces métaphores ne va pas de soi; elle est fréquemment précédée par des essais d'intégration ou des tentatives de discussion critiques comme on le voit à travers les usages conceptuels du " corps social» qui reposent parfois sur des oppositions explicites (Durkheim et Worms, Schäffle et Weber). L'autonomisation progressive des méthodes sociologiques, amorcée au cours du XIX ${ }^{\text {e }}$ siècle, n'est pas linéaire, mais elle concourt néanmoins à la dévaluation de certains modes d'appréhension (diffusionnismes psychologiques, finalismes métaphysiques, causalismes juridiques et naturalistes) concurrencés par de nouvelles démarches explicatives. Un regard dessillé peut être à nouveau projeté sur d'anciens terrains: l'analyse des phénomènes de rumeur passe de la psychopathologie des masses à l'étude des sociabilités ordinaires, de la philosophie du complot à l'examen circonstancié des modes de construction sociale de l'opinion ("Rumeur», Philippe Aldrin); l'analyse des logiques de formation des identités invalide les présupposés naturalistes, transhistoriques, continuistes, par un recours à des interprétations plurielles (interactionniste, constructiviste, historique) portant sur des déplacements biographiques, des fictions et des récits édifiants, des activités de performation sociale (politiques, artistiques, savantes) ("Identité », Annie Collovald) ; la sociologie des pratiques et des identités sexuées réinvestit des postures dénaturalisantes d'inspirations différentes (Margaret Mead, Foucault, Beauvoir) pour définir une nouvelle formule anti-essentialiste ("Genre », Christine Guionnet).

L'autonomisation des méthodes sociologiques n'est pas réductible à l'émergence de nouveaux principes d'intelligibilité du monde social. Elle renvoie à des dynamiques sociales de professionnalisation et de complexification du travail sociologique qui ont des effets immédiats sur le fonctionnement routinier de la discipline. Celle-ci intègre, au moins à partir du dernier tiers du XIX ${ }^{e}$ siècle, des phénomènes de concurrence intellectuelle et une différenciation du savoir sociologique ("Sociologie», Louis Pinto, Frédéric Lebaron, Claude Dubar). La circulation transnationale des modèles et des théories, la licitation du pluralisme cognitif, la formation de groupes et de sous-espaces disciplinaires expliquent en partie que des concurrences interprétatives se cristallisent autour des mêmes types de phénomènes ("Égalité et inégalité sociales ", Raymond Boudon; "Éducation », François Dubet). Les tensions autour de polarités héritées (quantitatif/qualitatif, holisme/individualisme, etc.) du bon usage de la critique sociale ou de l'expertise, des différentes représentations sociologiques (structurales, interactionnistes, constructivistes, etc.) des collectifs, imposent également des reconversions conceptuelles, des essais d'intégration de legs théoriques et empiriques controversés. En témoignent les réévaluations successives du concept de "classes sociales " qui mettent désormais en perspective, par un allongement qualitatif (historique, sociologique) du questionnaire marxiste, la construction relationnelle des groupes et les formes de domination (culturelle, politique, économique) à travers lesquelles se manifeste leur autorité ("Classes sociales", Gérard Mauger). Un grand nombre d'oppositions théoriques mises en relief par les contributeurs trouvent leurs raisons d'être dans des dynamiques endogènes à des sousdisciplines ou des groupes académiques, et dans des processus de dévaluation et de requalification des modèles et des styles sociologiques. On remarque ainsi des oppositions explicites entre les stratégies d'intégration des traditions sociologiques. Elles oscillent entre des entreprises d'intégration logique des héritages savants qui contribuent à la mise en forme de modèles

1. Sur les langages de la vision organique dans les sciences humaines et sociales, voir le classique de Judith Schlanger, Les Métaphores de l'organisme, Paris, Vrin, 1971. 
d'explication transposables sur des terrains différents ("Art, consommation culturelle ", Pierre Bourdieu), et des tentatives de comparaison et de neutralisation des traditions sociologiques qui donnent lieu à des régimes d'explication plurielle ("Art ", Nathalie Heinich). D'autres oppositions renvoient à la dimension directement polémique du travail sociologique notamment lorsque sont en jeu des définitions concurrentielles des phénomènes d'actualité et des définitions antinomiques du rapport du sociologue à l'actualité, comme le montre par exemple l'émergence d'une position de "diagnosticien du quotidien ", ou au contraire une critique du prophétisme sociologique $^{2}$, à propos du phénomène des massmedias ("Culture de masse", Edgar Morin). On peut encore souligner la présence d'entreprises de disqualification de modèles théoriques qui n'enregistrent des gains de connaissance qu'au prix de réformes internes, au risque d'apparaître aussi peu cohérentes que cumulatives («Systémisme», Michel Lallement). Il va de soi que l'amplification d'une critique des postures savantes (intellectualisme, objectivisme, subjectivisme) dans le sillage des travaux de Pierre Bourdieu a participé, en particulier au cours des années 1970, à la dévaluation de ces modèles théoriques ${ }^{3}$, alors que dans le même temps se consolidaient des répertoires d'analyse fondés sur un travail de généralisation contrôlée de recherches empiriques et collectives. Les effets conjugués de l'institutionnalisation et de la circulation de ces nouvelles « sociologies de la pratique» ont engagé à leur tour une "critique interne", entre reprise théorique et écart empirique, sur des terrains transversaux et stratégiques ("Socialisation", Bernard Lahire). Ils ont également favorisé la mise en place de modèles transactionnels modernisant des traditions passées ou à dépasser (systémisme, fonctionnalisme) ("Niklas Luhmann », Jean Clam) et intégrant les apports des théories de la pratique (les sociologies dites constructivistes) pour articuler sur un autre mode les phénomènes biographiques, cognitifs et systémiques ("Anthony Giddens", Antonin Cohen).

Le développement d'un répertoire d'analyse relationnel et historicisant au cours des dernières décennies a sans doute participé, plus que d'autres renouvellements de problématiques, à l'orientation du travail sociologique. C'est ce que suggèrent nombre de notices qui évoquent la place désormais centrale de l'analyse sociogénétique des groupes et des espaces sociaux. L'examen des processus de professionnalisation et d'autonomisation de collectifs plus ou moins institutionnalisés (journalistes, intellectuels, chômeurs, professionnels de la politique, etc.) a, de ce point de vue, l'avantage de remettre en perspective les différentes propriétés des groupes sociaux à partir des histoires complexes dont ils sont en partie le produit ("Presse", Erik Neveu; "Partis politiques", Éric Phélippeau). Il permet non seulement d'échapper aux ethnocentrismes des univers lettrés par un retour et un recours à l'histoire, mais aussi de restituer les logiques sociales de capitalisation de l'histoire par des formes collectives contemporaines (groupes, institutions, champs sociaux) ("Structuralisme génétique », Rémi Lenoir ; «Sociologie historique», Laurent Willemez). Aucune recherche n'illustre mieux ce redéploiement sociologique sur des terrains traditionnellement investis par des historiens, que la sociologie des producteurs et des biens intellectuels. Alors que l'historiographie traditionnelle, en particulier l'histoire des idées, recouvre sous le label transhistorique d'intellectuels des groupes affectés par des transformations structurelles et diachroniques profondes, la sociologie des intellectuels tend au contraire à rendre compte de l'historicité de cette

2. On renvoie pour mémoire à Pierre Bourdieu $\&$ Jean-Claude Passeron, "Sociologues des mythologies et mythologies de sociologues ", Les Temps Modernes, 1963, 211 : 998-1021.

3. Sur cette critique et cette crise de la "grande théorie" politique, voir Bernard Lacroix, "Systémisme ou systé-mystification ", Cahiers internationaux de sociologie, 1975, 58 : 97-122. 
catégorie, au double sens de catégorie verbale et d'ensemble social. Sont ainsi examinées les différentes formes et les différentes périodes d'autonomisation de la catégorie d'intellectuels, les luttes de définition dont elle est le lieu et l'enjeu (définition des frontières du groupe, définition des prises de position légitimes au sein du groupe), l'évolution de sa morphologie ou de sa composition sociale, de ses modes d'objectivation (institutionnalisation, spécialisation, professionnalisation, internationalisation, etc.), et de ses rapports structurels avec d'autres espaces sociaux (champs économique et politique) («Intellectuel », Gisèle Sapiro). La transposition de cette problématique sur d'autres terrains fait apparaître plusieurs régularités : le caractère fondamental du rapport des groupes à l'État qui fonctionne généralement comme un espace de redistribution et de légitimation des ressources et de l'autorité revendiquées par ces groupes, et la permanence de l'enjeu, pour une part politique, que représente pour eux leur autonomie sociale ("État», Hervé Fayat; «Professions ", Jean-Michel Eymeri). On peut ainsi relever l'importance des processus de construction politique des enjeux collectifs à travers des exemples aussi divers que l'édification de la nation par des groupes élitaires (spécialistes de la rationalisation politique, intellectuels d'État, élites culturelles) ("Nation», Anne-Marie Thiesse), l'annexion et la valorisation de certains biens culturels par des politiques publiques ("Culture», Vincent Dubois), ou la promotion politique des technologies de domestication de l'" opinion " ("Opinion publique ", Brigitte Gaïti). Il serait faux de croire qu'en France cette sociologie des institutions et des groupes repose uniquement sur des références théoriques nationales. Elle tend également à discuter, voire à consacrer, d'autres approches de ces phénomènes collectifs qui se présentent aussi sous l'aspect de mouvements ou de conflits sociaux, de productions normatives ou d'organisations en réseaux. Il faut sans doute signaler de ce point de vue l'intérêt de la recherche sociologique contemporaine pour les théories néo-institutionnalistes des ensembles organisés ("Économie », Frédéric Lebaron ; «Institution », Delphine Dulong), pour la sociologie comparée des processus de construction et de transformation de l'État (Tilly, Skocpol, Barrington Moore, etc. $)^{4}$, pour la sociologie politique des groupes professionnels et des procédures d'officialisation, d'appropriation politiques des savoirs professionnels qui articule les apports de l'interactionnisme aux sociologies classiques de la bureaucratie (Weber, Schumpeter $)^{5}$.

Sans se réduire à une série d'objets, de méthodes ou de terrains nouveaux, la systématisation des principes de l'analyse réflexive a engagé des transformations et des investissements durables en sociologie : une extension de la critique des impensés qui structurent la relation du sociologue à ses objets, et une nouvelle dynamique de recherche autour de la sociologie de la connaissance sociologique. Le passage d'une critique des objectivismes savants en tant que "préalable épistémologique " ${ }^{6}$ à un ensemble de recherches positives sur le travail savant (histoire sociale des disciplines et des savoirs académiques) constitue l'un des récents développements de la sociologie dont font état plusieurs notices. Le travail de la réflexivité, s'il est bien sûr constitutif de la démarche sociologique elle-même, a pris au cours des dernières années plusieurs directions (la spécification d'une sociologie de la science, l'analyse de la genèse des sciences humaines et sociales, la critique

4. Voir l'ensemble des contributions rassemblées dans Theda Skocpol, ed., Vision and Method in Historical Sociology, Cambridge-New York, Cambridge University Press, 1984.

5. Pour une présentation générale et éclairante, voir Eliot Freidson, "Formal Knowledge, Power and the Professions", in Professional Powers. A Study of the Institutionalization of Formal Knowledge, Chicago, University of Chicago Press, 1986: 1-19.

6. Pierre Bourdieu, Jean-Claude Chamboredon \& Jean-Claude Passeron, Le Métier de sociologue. Préalables épistémologiques, Paris-La Haye, Mouton, 1973: 29 sqq. 
épistémologique des sciences d'État, l'histoire sociale des inventions et des concepts savants). Les études relatives aux institutions et aux pratiques scientifiques mettent d'abord en évidence les différents cadres théoriques à l'œuvre dans la sociologie des sciences : l'analyse des normativités institutionnelles (R. K. Merton), des contingences sociales (intérêts, ressources, contextes) et des processus de construction de la science (de T. Kuhn au " programme fort »), l'étude discursive des controverses scientifiques et l'ethnographie des laboratoires, la sociologie structurale de la science et des espaces scientifiques ("Sciences», Yves Gingras). On assiste d'autre part à une historisation de la sociologie de la sociologie comme le montrent en particulier la sociologie des innovations, des traditions et des mises en tradition sociologiques ("École de Chicago ", Christian Topalov), l'analyse critique des usages sociaux et des méthodes des enquêtes politologiques ("Sondages d'opinion ", Alain Garrigou), l'étude des trajectoires de concepts sociologiques ("Suicide ", Philippe Riutort), la recontextualisation de catégories sociologiques mythifiées ("Neutralité axiologique», Isabelle Kalinowski), l'analyse des entreprises rétrospectives de fondation disciplinaire et des transferts internationaux de connaissances (les usages d'André Siegfried, l'importation des «sciences" de l'opinion : «Sociologie électorale ", Patrick Lehingue). Ces contributions présentent non seulement des usages de labels, de concepts, de théories savantes, mais elles proposent aussi une esquisse des intérêts spécifiques qui sont au fondement de ces usages savants. Elles montrent l'intérêt méthodologique d'une connaissance des intérêts à la connaissance qui permet à la fois de ressaisir les ventriloquies sociales du discours sociologique et les inconscients épistémologiques sur lesquels se fondent en partie les traditions sociologiques.

Une attention aux orientations théoriques les plus récentes et un pluralisme de rigueur qui accorde également à d'autres traditions sociologiques (École de Francfort, École sociologique du droit, théories de l'acteur rationnel, sociologie des mobilisations, etc.), à des méthodes d'analyse, notamment statistiques et quantitatives (tableaux de contingence, méthodes géométriques, etc.), et à des cadres théoriques réactualisés (théorie des réseaux, ethnométhodologie, etc.) la place qui leur revient, font du Dictionnaire de sociologie un volume un peu plus prometteur que ne le suggère son titre. Il s'agit bien davantage d'un dictionnaire de la sociologie, si l'on reconnaît avec les auteurs que celle-ci n'a rien d'une science achevée et unitaire.

Xavier Landrin 
MON NAIE

\author{
L'Argent des anthropologues, la monnaie des économistes \\ Paris, L'Harmattan, 2008, 320 p., notes bibliogr. \\ («Questions contemporaines. Globalisation et sciences »).
}

$\mathrm{N}$ OUVELLE tentative de faire dialoguer les disciplines sur le thème de la monnaie, ce travail collectif rassemble les contributions d'économistes, d'anthropologues et de sociologues. Douze articles concis et clairs se concentrent sur la grande diversité des usages et la construction symbolique de la confiance et de la légitimité de la monnaie.

Parue dix ans plus tôt, La Monnaie souveraine ${ }^{1}$ comportait déjà des éléments intéressants de réflexion commune, entre économistes et anthropologues, sur l'origine de la monnaie, sa teneur politique, rituelle et sacrificielle. Y avaient participé, entre autres, Mark Rogin Anspach, Charles Malamoud et Daniel de Coppet. L'initiative était partie d'économistes appartenant à l'École de la régulation, intéressés par les thèses d'obédience girardienne, convaincus que la monnaie, en tant que signe d'autorité et de lien collectif, préexiste à l'échange marchand, et qu'elle naît des dettes contractées non horizontalement, mais verticalement avec les puissances souveraines. Cet ouvrage avait provoqué de nombreuses réactions des anthropologues dont un numéro spécial de L'Homme ${ }^{2}$. Dans L'Argent des anthropologues, l'article de Jean-Michel Servet, Bruno Théret \& Zeynep Yildirim revient utilement sur ces critiques de façon très claire. Les auteurs commencent par réitérer l'hypothèse d'une universalité d'ordre anthropologique de l'existence de la monnaie; pour eux, celle-ci ne se limite pas aux fonctions de compte et de paiement, même si ces fonctions peuvent être considérées comme un dénominateur commun des monnaies marchandes et de la grande diversité des monnaies cérémonielles, primitives ou exotiques. Toutes sont des « instruments de quantification, de mise en circulation et de totalisation de ce qui est reconnu comme valeur dans une société. Elles transforment des obligations en dettes et des droits en créances» (p. 176).

Trois niveaux de difficulté dans la démarche de cet ouvrage peuvent être distingués. En premier lieu, la complexité et la richesse d'un objet d'étude à double face, dont Keith Hart montre bien qu'il est à la fois État et marché, instantanéité et mémoire, objet et idée, coercition et liberté, et qu'il constitue un défi pour les méthodes et les théories de l'anthropologie postcoloniale. En deuxième lieu, les terminologies et les usages lexicaux de disciplines différentes. Pepita Ould-Ahmed, dans l'introduction, reprend en particulier les nuances existant entre l'argent-stock, la

1. Michel Aglietta \& André Orléan, eds, La Monnaie souveraine, Paris, Odile Jacob, 1998. 2. L'Homme, 2002, 162 : Questions de monnaie. 
monnaie-flux, l'argent étant un sousensemble de la monnaie pour les économistes, quand cette dernière est une sous-catégorie de l'argent pour les anthropologues. Or, ce livre prouve que des économistes hétérodoxes et des anthropologues peuvent élaborer des modes convergents d'interprétation des phénomènes monétaires, au delà de l'autoréférence des jargons disciplinaires. Un bon exemple est la notion de cloisonnement entre usages monétaires: bien que les anthropologues préfèrent les notions d'usages, de pratiques, de sphères d'échanges, quant les économistes parlent, eux, de fongibilité (Jérôme Blanc), tous les articles sur la segmentation monétaire montrent que la monnaie valide des paroles et des liens sociaux. Elle est un «opérateur communicatif " chez les Yukuna décrits par Laurent Fontaine, elle valide « le pacte avec le diable » des mineurs de Potosí décrits par Pascale Absi, ou encore les statuts sociaux montrés avec ostentation lors des mariages au Caire (Nicolas Puig) ; elle est au cœur de la lutte pour le pouvoir financier au sein du couple chinois (assuré depuis longtemps par la cassette privée des épouses) dans le récit très enlevé de Thierry Pairault; elle dévoile l'attitude de "normalisation " paternaliste envers le pauvre de la part des banquiers (Gilles Lazuech et Pascale Moulévrier).

Sur l'autre axe du livre, celui de la légitimité, on voit comment l'optique macro-économique croisée avec les outils anthropologiques sur les symboliques permettent ensemble de comprendre comment certains régimes monétaires favorisent certains groupes sociaux au détriment d'autres, ou comment les mythes monétaires interagissent avec le conflit distributif: Jaime Marques-Pereira décrypte ainsi les récents bouleversements argentins et brésiliens ; Ludovic Desmedt reprend les choix de société aux origines de la naissance du dollar aux États-Unis, et Jean-François Ponsot la complexe configuration sociale et symbolique menant à l'acceptation du dollar en Équateur. Enfin, Laurent Bazin montre comment les options économiques et politiques de l'État ouzbek rendent la relation de travail arbitraire et oppressive envers les ouvriers d'une usine de filage et de chantiers de construction.

En dernier lieu, la difficulté de l'interdisciplinarité repose sur la perspective d'analyse et le problème comparatif. Pepita Ould-Ahmed décrit comment la monnaie est plutôt décrite " en régime" pour les anthropologues, sa valeur étant inscrite dans la particularité de chaque système social, tandis que les économistes hétérodoxes recherchent de nouvelles théories de la nature de la monnaie en étudiant des moments de genèse, de crise et de grande réforme, afin de montrer l'importance du politique dans la formation et la circulation de la monnaie. C'est sur ce point que demeure certainement un hiatus entre les deux disciplines: la possibilité ou non d'élaborer un faisceau de caractéristiques universelles qui iraient au-delà du simple rejet de la théorie orthodoxe de la «neutralité " de la monnaie et de la définition mentionnée plus haut. Mais peut-être ces différentes focales font-elles la richesse $\mathrm{du}$ kaléidoscope unidisciplinaire (OuldAhmed) nécessaire à l'étude de cet objet complexe. On ne peut donc que souhaiter que ce genre d'effort interdisciplinaire soit poursuivi. 


\section{SPORT\& TOURISME}

\section{Diffusion des sports et impérialisme anglo-saxon. De l'histoire événementielle à l'anthropologie}

Sébastien Darbon

Paris, Éd. de la Maison des sciences de l'homme, 2008, 369 p., bibl., index, ill.

$S$

ÉBASTIEN DARBOn livre ici un travail sur la diffusion géographique d'une invention moderne : le sport. Les travaux véritablement anthropologiques sur les pratiques sportives ne sont pas légion. Plus rares encore sont ceux consacrés à la diffusion géographique des sports, bien que celle-ci introduise, au même titre que pour d'autres productions culturelles, " aux questions de la diversité culturelle (entre pays diffuseur et pays d'accueil), des processus hégémoniques ou impérialistes, ou des voies complexes par lesquelles se construisent et s'expriment les revendications identitaires " (p. 303).

Louvrage est organisé en huit chapitres que complètent une bibliographie, un index et un cahier d'illustrations. Les trois premiers chapitres traitent des foyers d'origine: l'Angleterre, les États-Unis et leurs "prolongements impériaux ". Les quatre suivants s'attachent à la diffusion dans les colonies de peuplement : le sous-continent indien, la Caraïbe, et enfin l'Argentine et le Japon. Le dernier chapitre, qui fait office de conclusion, questionne les relations entre la démarche historique et la démarche anthropologique, et met au jour, dans une perspective théorique, les éléments à mobiliser pour expliquer "pourquoi les choses se sont passées ainsi et pas autrement" (p. 338).
Dans la lignée des sociologues Elias et Dunning, le sport est envisagé ici, non comme une continuité des jeux traditionnels, mais comme une rupture avec eux. Cette innovation, due à l'Angleterre du milieu du XVIII ${ }^{\mathrm{e}}$ siècle, se caractérise par « une extraordinaire potentialité d'universalité en essaimant dans le monde entier "essaimage dans lequel le fait colonial a joué un rôle déterminant (p. 4). Une analyse plus attentive montre pourtant que la répartition géographique des pratiques sportives est très inégale. Il s'agit donc de comprendre les raisons qui ont fait que tel ou tel sport a pu être accepté ou refusé dans tel ou tel pays, et d'expliquer les modifications que le contact avec un contexte culturel très différent, celui du pays d'accueil, a souvent entraîné. Pour ce faire, Sébastien Darbon mobilise les connaissances déjà produites, principalement des travaux historiques anglo-saxons. Compte tenu de l'ampleur du sujet, il se cantonne à un petit nombre de pays choisis pour leur importance ou leur situation particulière dans le processus de colonisation, privilégie les sports collectifs de balle ou de ballon, et situe son étude plus particulièrement dans la seconde moitié du XIXe siècle et la première moitié du XXe.

Le sport naît de la conception originale des relations entre le corps et l'esprit - l'idéologie de la «chrétienté musculaire» - 
propre à la culture victorienne. Le système éducatif anglais (les public schools et les collèges universitaires, établissements réservés à l'élite sociale), la religion et la littérature participèrent, chacun à leur manière et malgré des oppositions, à l'émergence de cette idéologie, le premier voyant dans les exercices physiques un moyen efficace de contrôler des élèves souvent en rébellion contre l'institution, la deuxième inscrivant le corps du chrétien dans l'action - «le corps est au service du monde, il faut donc le rendre de fort " (p. 39) -, la troisième usant de façon extensive des analogies sportives. Bien que beaucoup de pratiques sportives soient concernées, la trilogie cricket/soccer/rugby s'imposera " comme le fer de lance de la pénétration de la chrétienté musculaire" (pp. 11-12), diffusant peu à peu le modèle du fair-play dans toute la société anglaise.

Le phénomène sportif aux États-Unis suit les mêmes voies de développement qu'en Grande-Bretagne : système éducatif, religion, littérature. Il se caractérise par l'apparition de pratiques qui, soit dérivent de jeux anglais (base-ball, football), soit sont inventées sur place (volley-ball, basketball) par un protestantisme évangélique investissant l'homme sportif de "la lourde tâche de sauver la société contre elle-même " (p. 57). Les sports collectifs américains se distinguent par une spécialisation des tâches, une rationalisation où la dimension ludique cède place à l'application systématique de principes en vigueur dans le monde industriel (p. 73). La victoire y importe davantage que la manière de gagner.

Les travaux historiographiques permettent de dresser la carte de la répartition des sports façonnée par l'impérialisme anglosaxon dans ses zones d'influence. Ainsi en Australie, alors que le cricket devient rapidement un sport spectacle, que le rugby à XV, amateur, et le rugby à XIII, professionnel, s'opposent, un sport national, le footie, émerge ; le soccer quant à lui attire les immigrants tardifs et prend une dimension communautaire ; enfin l'influence américaine se fait sentir à partir de la seconde moitié du XIXe siècle avec l'introduction du base-ball. En Nouvelle-Zélande, le cricket, considéré comme sport national jusque dans les années 1890, est rapidement détrôné par le rugby. En Afrique du Sud, les Afrikaners adoptent le rugby; les Africains, les métis et les Indiens s'emparent du soccer pour en faire le sport le plus pratiqué, tandis que le cricket reste la chasse gardée des Britanniques malgré l'existence, à une époque, d'un cricket de "non-Blancs ». En Inde, le cricket, stimulé par la concurrence intercommunautaire, triomphe, au point qu'il semble aux Indiens plus indien qu'anglais; à la marge, se développe le soccer. Dans les Antilles britanniques, le cricket et le basket-ball s'imposent alors que le base-ball domine dans la Caraïbe hispano-américaine. L'Argentine est séduite par l'essentiel des sports anglais: rugby et polo (rapporté d'Inde du Nord par les officiers britanniques) chez les élites, football (fûtbol) dans les couches populaires. Au Japon, le baseball connaît un succès prodigieux.

Au terme de cette description, Sébastien Darbon tire les enseignements de la littérature historique explorée. Cette diffusion des sports ne répond pas à «l'application d'un principe général systématique, réfléchi et volontariste qui s'appuierait sur une conception du sport comme l'instrument d'une imposition de l'État colonial " (p. 306). Les Britanniques, par exemple, souhaitaient avant tout jouer entre eux et préserver ainsi leurs valeurs culturelles de référence. Ils se montrèrent souvent réticents, dans un premier temps, à laisser pratiquer leurs sports par des colonisés qui remportèrent parfois de haute lutte le droit de le faire. Cette réticence s'estompa plus tard devant la qualité des joueurs locaux ou devant la place prise par le sport dans le jeu politique colonial. Les Américains, eux, persuadés des idéaux qu'ils rattachaient à leurs sports - démocratie, liberté, modernité -, n'émirent aucune réserve à leur diffusion. Pour l'auteur, l'historiographie du sport, essentiellement fondée sur des monographies privilégiant "le particulier au détriment de la recherche de régularités ou 
d'invariances" (p. 313, italiques de Sébastien Darbon), se révèle utile pour saisir comment s'est effectuée cette diffusion géographique, mais n'apporte que peu d'éléments quant au pourquoi de ses itinéraires et de ses rythmes. Pour expliquer pourquoi un sport a suscité un fort engouement (ou a été rejeté), il convient, selon Sébastien Darbon, de réaliser une analyse fine de ses propriétés formelles - l'ensemble de ses règles du jeu - et de mener une étude ethnographique approfondie des traits culturels de la société concernée, de manière à saisir la "rencontre singulière entre un certain sport et un contexte culturel spécifique» (p. 339). L'analyse de l'adoption du cricket par les Indiens et de leur refus du rugby lui permet de mettre en évidence la pertinence d'une telle démarche.

Très documentée, cette "aventure » des pratiques sportives, à l'édition soignée malgré quelques scories, ne manquera pas de séduire un lectorat érudit, notamment par les descriptions de certains personnages hauts en couleurs. Ce travail viendra également combler un vide sur les rayons des bibliothèques universitaires, notamment par ses aspects pédagogiques et par sa réflexion critique des sources explorées (en particulier, le chapitre VIII qui fait la synthèse des enseignements de l'approche historique en anthropologie est très instructif). L'anthropologue, quant à lui, restera un peu sur sa faim. Car Sébastien Darbon aiguise son appétit en le convainquant de la pertinence d'une approche anthropologique pour explorer le phénomène de la diffusion des sports, puis il tourne court au moment où l'affaire pourrait devenir à proprement parler anthropologique. On regrette notamment que la piste des transformations induites par les frottements d'une culture sportive importée avec le contextuel culturel d'accueil n'ait pas été suivie plus longtemps. L'indianisation ou la créolisation du cricket, ou encore la "japonisation " du baseball constituent des réinterprétations qui auraient pu être abordées avec profit sous l'angle des processus d'acculturation. Enfin, on s'étonne que l'auteur, tout à la construction de sa propre "perspective théorique" (p. 339), n'évoque pas davantage les théories de la diffusion, leurs heurs et leurs malheurs. 
C

E NUMÉRO DES Actes de la recherche en sciences sociales est consacré à un thème, le tourisme, encore largement délaissé par les chercheurs. S'il est abordé par les sociologues, c'est dans le cadre de domaines d'investigation considérés comme plus légitimes, tels que l'environnement ou la famille. L'article introductif de Bertrand Réau et Franck Poupeau analyse cette relative carence. Le tourisme ne trouverait réellement sa place aujourd'hui que dans des recherches sur la mondialisation dominées par la problématique du développement durable et par la «littérature grise »; il s'apparenterait ainsi " moins à un objet de recherche qu'à un "label politique”" (p. 6). Le peu de légitimité de cet objet dans l'espace académique français et la faiblesse des financements de recherches fondamentales auraient contribué à renforcer la perméabilité de la frontière entre consulting, recherche appliquée et recherche fondamentale: "Se noue ainsi une interdépendance entre les chercheurs et l'univers professionnel du tourisme: les institutions publiques transposent des méthodes et des grilles de lecture du secteur privé; les professionnels du tourisme s'inspirent, en retour, des analyses proposées par les universitaires et les rapports d'organisations publiques (souvent produits par ces mêmes universitaires)»(p. 9). Cette interdépendance contribue à forger une relation paradoxale du tourisme à l'économie : "d'un côté, il est courant de valoriser le marché touristique; de l'autre, les touristes et professionnels du tourisme coproduisent un déni des caractéristiques marchandes des services et une relation enchantée au monde social» (p. 10). L'analyse de cette relation paradoxale constitue le fil conducteur de ce numéro qui «vise ainsi à étudier les conditions sociales de l'économie du tourisme auxquelles participent différents groupes sociaux: associations, États, organismes internationaux, opérateurs privés, etc. " (id.).

Les articles constituant le dossier éclairent ainsi le rapport paradoxal à l'économie de diverses formes contemporaines de tourisme: accueil en chambres d'hôte, tourisme dit "solidaire", tourisme religieux, tourisme sportif... Christophe Giraud analyse la "mise en scène de l'accueil marchand en chambres d'hôte " où il s'agit de "recevoir le touriste en ami" (p. 15). L'oxymore "accueil marchand" permet de caractériser la double nature d'une activité consistant à héberger des "hôtes payants"; si l'accueil ne peut être perçu comme seulement marchand, il ne doit pas non plus être appréhendé comme exclusivement amical par des touristes devant régler la note à leur départ. La relation instaurée ne doit pas non plus être perçue sur le mode d'une relation ancillaire, les propriétaires dirigeant leur maison et restant des égaux des touristes qu'ils servent. "Ces trois grands modèles de relations (marchande, amicale, ancillaire) constituent les cadres d'interaction qui permettent aux touristes comme aux prestataires de définir la situation" (pp. 15-16). L'auteur analyse le travail des propriétaires jouant sur ces cadres d'interaction et souligne combien, lors même que la dynamique de la relation amène à euphémiser la dimension marchande, celle-ci reste très présente.

Nadège Chabloz étudie "Les rencontres paradoxales du "tourisme solidaire" " (pp. 32-47) en présentant les résultats d'une observation participante dans un village du Burkina Faso. S'il promet une rencontre "authentique" entre les touristes et les villageois visités, ce type de contact repose en fait sur des malentendus provenant largement de la méconnaissance réciproque 
et renforcés par les discours liés au tourisme. L'association organisatrice, en effet, mais aussi les villageois et les touristes construisent l' "illusion" de la rencontre: les dirigeants de l'organisation, en ce qu'ils sont engagés dans la double mission de faire du développement et vendre des séjours, produisent des discours paradoxaux sources de malentendus, les villageois laissent croire aux touristes qu'ils sont intégrés tout en s'efforçant de les maintenir à distance, tandis que les touristes transforment l'objet de la rencontre en « objet de visite» (p. 45), ne se privant pas de photographier abondamment les autochtones. S'ajoute à cela le paradoxe d'un tourisme orienté vers le développement local mais jouant sur la volonté de s'immerger dans des pays préservés de la modernité.

L'analyse par Xavier Zunigo "Sur les ambiguïtés d'une pratique humanitaire et caritative à Calcutta » (pp. 102-109) révèle également les nombreux malentendus sur lesquels s'établissent les relations entre voyageurs et populations pauvres visitées. Les Missionnaires de la charité, ordre religieux fondé à Calcutta par Mère Teresa, attirent ainsi chaque année des centaines de bénévoles occidentaux de nationalités et obédiences religieuses contrastées sous le couvert d'une forme de tourisme atypique, humanitaire ; mais aucun de ces volontaires ne conçoit son séjour comme touristique. Un autre type de tourisme religieux, le "pèlerinage flottant " autour du mont Athos, présenté par Filareti Kotzi, est également révélateur de ces contradictions. Lauteur y étudie notamment l'osmose entre les caractères sacré et économique des souvenirs religieux vendus par les moines lors du pèlerinage.

Les voyages à l'étranger organisés pendant les années 1970 par une association de tourisme social, Tourisme \& Travail, liée aux comités d'entreprise de la CGT, sont pour leur part révélateurs du décalage existant entre le projet élaboré par une élite militante et sa réception par le public visé. C'est ce que montre l'article que Sylvain Pattieu consacre à cette expérience. Ce tourisme social s'inscrit dans un projet politique et syndical d'éducation populaire par les loisirs lié aux représentations du mouvement ouvrier et à l'expérience par l'élite porteuse du projet d'une promotion sociale par le militantisme. Cependant, les pratiques des personnels et vacanciers des centres restèrent largement tournées vers la distraction; ce relatif échec culturel contribua dans les années 1980 à une transformation de cette association de tourisme social en entreprise sociale de tourisme. À cet échec s'oppose, pourrait-on dire, le «bricolage réussi " (p. 68) auquel s'apparentent les clubs Méditerranée étudiés par Bertrand Réau. Ce dernier revient ainsi sur la naissance, entre 1948 et 1950 , de l'idée, alors novatrice, d'associer des activités sportives, d'animation, avec le transport, la restauration et l'hébergement. Il retrace les conditions sociales de l'invention, par certains membres de l'équipe de water-polo du Racing-Club de France et leurs proches, de cette « forme de vacances mettant en ouvre une conception hédoniste des loisirs fondée sur le jeu comme finalité et non plus comme moyen éducatif» (p. 68), convertissant ainsi leurs ressources sportives en capital économique.

Anne-Catherine Wagner, dans l'article qu' elle consacre à « La place du voyage dans la formation des élites » (pp. 58-65) rappelle combien le tourisme est une pratique socialement distinctive, qui s'inscrit dans une tradition, celle du "grand tour», et garde aujourd'hui une place centrale dans les pratiques éducatives des classes sociales supérieures, «soucieuses de transmettre des ressources internationales, comme l'atteste le fonctionnement des écoles bilingues ou internationales destinées à cette population» (p. 60). Nonobstant la diffusion des voyages de formation dans les classes supérieures et même dans une fraction des classes moyennes, ils conservent pour les héritiers des grandes familles leurs propriétés distinctives, souligne l'auteure en s'appuyant sur deux exemples à partir d'entretiens menés auprès de membres de la haute bourgeoisie: "Occasion d'expérimenter, sous une forme ludique, d'autres 
rôles sociaux que celui de grand bourgeois " (p. 64), le voyage préparerait « aux stratégies de distanciation exigées par l'occupation de positions multiples dans différents champs du pouvoir» (id.) tout en permettant «d'éprouver le capital international familial, au sens du réseau relationnel et des affaires de famille» (p. 65).

Riche, documenté, présentant de manière convaincante à partir d'études de cas diversifiées le rapport paradoxal que le tourisme, quelle qu'en soit la forme, entretient avec l'économie, ce numéro des Actes montre tout l'intérêt d'étudier les multiples facettes de cet objet longtemps négligé, en conciliant les regards du sociologue, de l'ethnologue, mais aussi de l'historien. Il se conclut opportunément par une lecture critique de Sylvain Pattieu de l'ouvrage de Daniel Roche, Humeurs vagabondes. De la circulation des hommes et de l'utilité des voyages (Paris, Fayard, 2003), dont la conclusion pourrait être transposée ici : «le regard n'en reste pas moins critique pour déceler dans les progrès de la mobilité à l'époque moderne la part d'ombre qui en subsiste et qui s'abrite parfois derrière la "civilisation des mœurs" " (p. 114).

Corinne Delmas 


\title{
A MÉ RIQ UES
}

\author{
Jean-Pierre Lavaud \& Isabelle Daillant, eds \\ La Catégorisation ethnique en Bolivie. \\ Labellisation officielle et sentiment d'appartenance \\ Paris, L'Harmattan, 2007, 294 p., bibl., fig., tabl., cartes.
}

C

et ouvrage collectif rassemble dix contributions consacrées à la catégorisation ethnique en Bolivie. Dans la première partie, les différentes études considèrent les procédures officielles de labellisation et de comptabilisation des groupes ethniques boliviens et, plus largement, de celles des Indiens. Il s'agit d'une présentation critique des catégorisations officielles, telles qu'elles apparaissent dans les documents administratifs. Dans la seconde partie, les contributions portent davantage sur la façon dont les catégorisations ethniques sont pensées et utilisées au quotidien dans trois contextes urbains ou villageois d'altitude : La Paz, Potosi et Mocomoco.

Dans son introduction, Jean-Pierre Lavaud insiste sur un point fondamental : les catégories traitées dans l'ouvrage sont à l'origine inspirées par des impératifs administratifs et utilisées pour les recensements des populations; en cela leur pertinence est d'emblée limitée dans la mesure où elles sont en premier lieu un instrument de gestion politique. C'est pourquoi les résultats des recensements doivent toujours être réinterprétés après avoir discuté les catégories. Parmi ces catégories de classement, les appellations officielles ethniques, culturelles ou raciales revêtent socialement la plus grande légitimité. À un autre niveau, toujours selon Jean-Pierre Lavaud, il est indispensable de contextualiser ces mécanismes officiels d'attribution en tenant compte de l'état social, économique et politique du pays à une période donnée; ce d'autant plus que, dans toutes les sociétés modernes, le contexte est toujours inégalitaire, quel que soit le critère de référence (économique, social, éducatif...). Ainsi, toute catégorisation se réfere à un classement tenant compte d'une échelle nécessairement discutable. Généralement, ces hiérarchisations sont d'ailleurs perçues comme plus ou moins légitimes selon les groupes, les contextes et les individus au sein des groupes.

Le premier essai de Daniel Dory explicite les différentes variations des localisations géographiques et des comptabilisations des groupes ethniques constatées dans les dénombrements effectués depuis 1830 . Il propose la construction d'une "géographie ethnoculturelle» tenant compte des positions et des relations de ces différents groupes ethniques, notamment des changements importants induits depuis un demi-siècle par l'ensemble des migrations, insistant ainsi sur le caractère dynamique de leur évolution. La contribution de Rosanna Barragán porte sur la répartition de la population bolivienne selon le critère distinctif de la race tel qu'il apparaissait dans les divers recensements nationaux du 
XIX ${ }^{\mathrm{e}}$ siècle, et plus particulièrement dans les résultats de celui de la ville de $\mathrm{La} \mathrm{Paz}$ effectué en 1889. À cette époque, la catégorie de métis était employée, avant d'être abandonnée lors du recensement de 1950 qui fait appel à des critères culturels pour distinguer les Indiens des non-Indiens. Rosanna Barragán analyse également l'articulation entre catégories sociales (l'emploi ou l'activité de l'acteur) et catégories dites raciales. Jean-Pierre Lavaud, quant à lui, montre bien à quel point la nature des taxinomies employées influe sur les résultats dans la comptabilisation de la population indienne. Des écarts importants apparaissent en effet selon les types de critères d'identification ethnique utilisés. En outre, les récents choix classificatoires répondent, pour une grande part, à des options politiques: ils s'inscrivent dans une logique d'identification d'ensembles spatiaux ethnoculturels avec, pour finalité, une tentative de mise en place de politiques locales différenciées dans une logique de discrimination positive.

Plus loin, les études de Véronique Marchand et Pascale Absi illustrent le caractère dynamique de l'usage quotidien du terme cholo (métis) : elles font en effet ressortir que ce terme à connotation péjorative est utilisé, dans un cas, pour désigner les commerçantes indiennes en ascension sociale à La Paz, mais ne l'est pas, dans un autre contexte, pour désigner - ou autodésigner - des mineurs des coopératives de Potosi qui sont pourtant généralement officiellement rattachés à ce même univers intermédiaire. Quant à Cécile Claudel, elle s'intéresse à la petite bourgeoisie locale, et plus particulièrement aux familles devenues vecinos lors de la réforme agraire de 1953, alors qu'elles étaient au préalable considérées comme mestizos ou cholos. Puis, Laurence Charlier montre les différentes formes d'identification que les personnes issues du nord du département de Potosi en migration sont amenées à exprimer durant leur parcours, selon les milieux et les personnes rencontrées. Enfin, Rosalía Martínez aborde elle aussi la question identitaire mais, cette fois, à partir de la thématique de la signature musicale, en décrivant l'évolution qui se dessine vers des formes musicales métissées, influencées par les mélodies diffusées par les radios locales et régies par des mécanismes particuliers.

Les deux dernières contributions traitent des indigènes des Basses-Terres tropicales. Isabelle Combès se consacre à l'ethnogenèse des Chiriguanos dispersés dans les provinces de Santa Cruz, Chuquisaca et Tarija. Il apparaît que le monde chiriguano - ou guarani - actuel n'a cessé de se transformer au gré des différents conflits tant internes qu'externes. Enfin, Isabelle Daillant examine les rapports stratégiques et circonstanciels d'identifications entre trois populations de la région de San Borja (Beni) - les indigènes chimane, les cambas (Blancs et métis locaux) et les migrants collas (Andins) -, rapports déterminés ou, pour le moins, conditionnés, par l'usage de la référence aux notions d'indianité et d'autochtonie.

Cet ouvrage offre donc des données à la fois contradictoires et complémentaires qui permettent une connaissance plus fine du lien entre sentiment d'appartenance et labellisation officielle en Bolivie, selon les époques. Cet apport est d'autant plus précieux que les recensements actuels ont abandonné les catégorisations ethniques intermédiaires au profit de types culturels ou ethniques premiers, et donc d'une lecture essentialiste du fait social.

Thomas Pierre 


\section{Ties That Bind.}

The Story of an Afro-Cherokee Family in Slavery and Freedom Berkeley-London, University of Californa Press, 2005, 306 p., bibl., index, ill., cartes.

\section{6}

LES RECONSTRUCTIONS imaginées du passé, intimement tissées aux connaissances historiques, peuvent nous aider " (p. 60) à approcher l'expérience subjective des acteurs : c'est ce qu'affirme Tiya Miles, maître de conférence à l'Université de Michigan. Cette méthodologie aurait de quoi surprendre si ne se trouvait au cœur du livre la question de la mémoire. Venue chercher le récit d'un "front commun [des Noirs et des Indiens] contre l'esclavage et le colonialisme»(p. XIII), l'auteure dut en réalité trouver le courage de maintenir le projet de sa recherche face à des résistances que leur caractère affectif rendait d'autant plus redoutables. Car c'est une évidence : Africains, Américains et peuples autochtones "se rappellent et imaginent le passé en des termes différents" (p. XIV). Pourtant, et le titre l'indique bien, "il y a des liens qui unissent »- pour le meilleur et pour le pire, est-on tenté de dire devant les rebondissements le plus souvent tragiques, mais pas toujours fatidiques, de cette histoire à la fois familiale et nationale, singulière et collective.

L'héroïne du livre est un de ces personnages généralement oubliés de l'histoire: une femme noire. Entrée au tournant du XVIII ${ }^{\mathrm{e}}$ siècle au service du guerrier cherokee Shoe Boots, elle devint sa compagne sans cesser d'être son esclave. S'appuyant sur des archives nombreuses en raison de la notoriété de Shoe Boots mais peu informatives sur ce que fut Doll, le livre pose deux questions principales (p. 4): comment les conceptions esclavagistes furent-elles absorbées ou rejetées par le système de parenté cherokee? Et quel est le lien entre émancipation des Noirs et souveraineté cherokee?

Le tout premier chapitre ("Captivity») retrace un premier concubinage du guerrier
Shoe Boots avec une adolescente blanche enlevée lors d'un raid et finalement restituée à sa famille. Doll n'est alors que l'un des deux esclaves africains dont dispose le couple. Par cette première mise au point, Tiya Miles fait un choix astucieux car il permet de voir, à travers le récit propagandiste relaté par les journaux locaux, la construction d'un idéal de femme blanche aux vertus civilisatrices. L'auteure prend soin de souligner également l'adhésion des Cherokee, soucieux de reconnaissance, à cet idéal féminin domestique dont le versant négatif, par une triangulaire bien connue du monde colonial, est occupé par la femme noire.

Après cette introduction relativement biographique, le chapitre "Slavery" fait le rappel de la coprésence afro-indienne, jusqu'au début du XVIII ${ }^{\mathrm{e}}$ siècle, sur les plantations du Sud, et de l'asile jusque-là généralement offert par les Cherokee aux fugitifs d'origine africaine. L'esclavage cherokee au départ peu coercitif devint, au tournant du XIX ${ }^{e}$ siècle, progressivement perméable aux préjugés et aux politiques anti Noirs et, à la fin de la Révolution américaine, démontre Tiya Miles, les Cherokee, contraints d'accepter l'aide gouvernementale, sont "poussés à revoir leur relation au monde» (p. 35). Ces transformations ne mettent cependant pas totalement fin à leur tradition d'hospitalité à l'égard des fugitifs : à leur façon, analyse l'auteure, les Cherokee "reproduisaient et résistaient simultanément au système esclavagiste euro-américain» (p. 33).

Le chapitre III ("Motherhood»), toujours attaché aux pas de la jeune esclave-concubine, permet d'aborder l'incidence fondamentale du système de parenté dans la réception de l'esclavage par les 
Cherokee. Dans une communauté où l'on est membre d'un clan par la mère, explique sans affiliation. L'inclusion de Doll dans la vie privée de Shoe Boots en même temps que son exclusion de la communauté cherokee sont finement décrites par des exemples tirés de la vie quotidienne : ainsi Doll mange-t-elle à la table de son compagnon, non avec les autres femmes.

Le chapitre IV ( "Property») traite ensuite de la question de la propriété, jusque-là absente de l'univers cherokee, et de son lien direct avec l'exploitation coloniale et la définition des « races". Le programme politique visant à "civiliser" les Indiens les amène en effet progressivement, vers la fin du XVIII ${ }^{\mathrm{e}}$ siècle, à envisager la propriété, et notamment celle des esclaves. Cette section ouvre également une réflexion comparative stimulante sur les Creek qui, quand les esclaves sont peu nombreux, leur autorisent vie familiale autonome, libre déplacement, voire propriété. Cependant, chez les Creek comme chez les Cherokee, apparaît une stratification de classe, aboutissant à une guerre civile, la Redsticks Rebellion.

Le chapitre suivant ("Christianity») retrace l'arrivée, dans les années 1820, dans le bourg de Shoe Boots, d'une mission évangélique abolitionniste. Les Noirs y sont non seulement acceptés mais leur connaissance de l'anglais et des techniques européennes fait d'eux des "médiateurs culturels » reconnus et appréciés (p. 96).

Au chapitre VI («Nationhood»), est analysée la création, au début du XIX ${ }^{\mathrm{e}}$ siècle, d'un gouvernement cherokee plus restreint, plus jeune et plus blanc. L'application de la loi passe ainsi des mains des clans à celles des cours de justice; femmes et Afrodescendants sont exclus de la participation au pouvoir politique. Une sorte de "Code noir » est esquissé. Mais 1824 est aussi l'année où Shoe Boots demande - et obtient l'émancipation et la reconnaissance en tant que Cherokee des enfants nés de Doll, utilisant pour cela, dans sa rhétorique, la symbolique puissante du "sang" (principe maternel) et de l'«os» (principe paternel).

À la suite de la découverte de l'or en territoire cherokee, apprend-on au chapitre VII ("Gold Rush»), l'État de Géorgie annule les lois cherokee. Après la mort de Shoe Boots, Doll et ses enfants, pourtant émancipés, sont pris en chasse; un neveu de Shoe Boots choisit alors « de définir Doll et les enfants (de son oncle) comme biens plutôt que comme parents" (p. 136). En effet, écrit Tiya Miles, « les peuples autochtones du Sud-Est voyaient dans la parenté, non dans la liberté, l'antithèse de l'esclavage. Dans l'esprit d'un Cherokee, la parenté disait l'humanité, et l'humanité signifiait la liberté»(p. 142). C'est sans doute là le point central du livre.

Déportés vers l'actuel Oklahoma (chapitre VIII, « Removal »), autrement dit vers l'Ouest, " une direction qui dans la culture cherokee est synonyme de mort et d'obscurité » (p. 152), les Cherokee se trouvent non seulement dépossédés de leurs biens (au profit de colons blancs), mais privés d'un paysage naturel vécu comme support d'enseignements moraux. Le chapitre IX ("Capture») montre ainsi comment, dans un contexte de pénurie " [sont intensifiées] les inégalités économiques " - alors que prospère une élite d'ascendance mixte eurocherokee, possesseurs ou marchands d'esclaves (p. 164). Avec le dernier chapitre («Freedom»), Doll, devenue inutile, est libérée, à l'âge de 70 ans. Elle est enfin officiellement reconnue comme la veuve de Shoe Boots.

L'épilogue ("Citizenship») suit les descendants du couple, premier de ce type à voir son union régularisée, et traite de la difficulté pour les Afro-Cherokee à être reconnus comme Cherokee lorsqu'ils le désirent, contrairement en cela aux métis euro-cherokee.

L'Appendice I traite de méthodologie : en tant que premier peuple autochtone à écrire sa langue, les Cherokee ont produit quantité de documents écrits et suscité une attention particulière de la part des universitaires. Pour l'auteure, il faut se garder d'y voir la possibilité d'une «transparence » (p. 209) et ne pas «accepter l'autoreprésentation de 
Cherokee particuliers comme vérité historique» (p. 208). Shoe Boots, rappelle-t-elle, incarnait à merveille l' "Indien romantique [...] dont l'image mythifiée était en train de devenir populaire" (p. 210).

L'Appendice II propose une discussion des termes de métissage: muste, mulatto, half-breed, Negro, Mixed Negro, jusqu'au récent Black Indian, qui pour l'auteure, ne dit pas grand-chose de la grande variété des situations autochtones. De même, s'interroge-t-elle, où placer le marqueur Afro ou
Black? Que dit-on alors de la priorité de l'un ou de l'autre dans l'expérience de celui qui passa toute sa vie en contexte indien?

Enfin l'Appendice III rend compte des erreurs répétées dans la compréhension comme dans la transcription des noms indiens: de ce point de vue, Doll comme Shoe Boots auront vu l'un et l'autre leur identité maintes fois déplacée...

Natacha Giafferi-Dombre 
L

E LIVRE que Catherine Clémentin-Ojha consacre aux chrétiens de l'Inde est à la fois bienvenu dans le contexte politique indien actuel, et stimulant pour l'anthropologie de l'Inde. Publiant en tant qu'indianiste, l'auteure prend néanmoins soin de clarifier les enjeux proprement indiens de son sujet, ce qui, ajouté à plusieurs cartes et tableaux récapitulatifs, un glossaire, une chronologie et un index, rend l'ouvrage stimulant audelà de l'anthropologie de l'Inde. Dans une première partie, l'auteure expose le « mode d'insertion [des chrétiens de l'Inde] dans la société indienne», en insistant sur leur situation légale et administrative. La seconde partie étudie le «legs du passé missionnaire », en soulignant la diversité interne à la communauté chrétienne indienne, et les stratégies évangélisatrices concurrentes, avant de conclure sur les processus plus ou moins récents d'indianisation du christianisme.

Le contexte indien dans lequel s'inscrit ce livre est, ironiquement, celui du nationalisme hindou qui sévit depuis une vingtaine d'années. Cette idéologie communautariste selon laquelle l'Inde serait exclusivement hindoue est portée par une branche politique (le BJP, parti du peuple indien), acteur clé de la politique nationale, mais aussi par une branche paramilitaire virulente (le RSS, association des volontaires nationaux). On ne s'étonnera donc pas que les chrétiens indiens, à l'instar des musulmans indiens, soient la cible d'attaques de plus en plus violentes, parfois tolérées par les autorités.

Pourtant, l'idéologie nationaliste hindoue est à la fois anti-occidentale, exigeant des adeptes de religions exogènes à l'Inde (christianisme et islam) qu'ils indianisent leurs fois et leurs pratiques et ancrée dans des schémas hérités de l'Occident, ce dont l'auteur parle peu. Ainsi la construction de l'hindouisme comme religion, la perception de son unité, sans parler du système des castes et du communautarisme, renvoientils également aux catégorisations du colonisateur. Plus concrètement, le prosélytisme hindou a notamment appris des missionnaires chrétiens l'importance d'investir dans l'éducation et la santé.

Quelques chiffres posent bien le problème: les $83 \%$ d'hindous que compte l'Inde seraient, à en croire les nationalistes, menacés par les... 2\% de chrétiens. S'agirait-il d'une minorité certes très faible mais à croissance exponentielle ? Non : le taux de chrétiens en Inde est stable depuis 1961, et le taux d'accroissement de leur population est même passé de 36\% (années 1960) à $17 \%$ (années 1980), ce que l'auteure explique notamment par l'éducation des femmes, l'urbanisation et la fin des 
conversions collectives. Serait-ce alors la forte visibilité de cette communauté chrétienne qui expliquerait le fantasme d'un danger pour l'Inde hindoue? Non plus : la communauté sikhe, avec une population légèrement moins nombreuse (19 millions pour 24 millions de chrétiens) est nettement plus visible. En outre, les chrétiens, au contraire des sikhs, ne disposent ni d'une région particulière où cristalliser leurs revendications et leur singularité, ni même d'institutions centrales capables de les fédérer: il a fallu attendre les violences hindoues à leur égard pour entendre une voix chrétienne unie.

Alors en quoi les hindouistes se sententils menacés? Sans doute parce que le christianisme, comme l'islam, est une religion à la fois exogène et prosélyte et qu'elle reste marquée par le sceau du colonialisme, donc par un processus inverse à l'indianisation souhaitée. Ainsi la population chrétienne de rite syrien du Kerala, présente bien avant la colonisation, cumulant l'intégration au système social local (en haut de la hiérarchie des castes) et l'absence de prosélytisme, n'a-t-elle pas souffert de discrimination. Mais ni l'arrivée des missionnaires ni l'histoire post-indépendance n'ont bouleversé l'équilibre socioreligieux indien. Et le panorama que brosse l'auteure de l'histoire des missions chrétiennes en Inde démontre qu'une foi exogène ne saurait y prendre racine, même après cinq siècles de colonisation, sans s'adapter à la société indienne.

Les nationalistes hindous semblent en fait surtout s'émouvoir de l'émergence d'une " théologie dalit [opprimés] " qui vise les plus défavorisés des Indiens, critiquant l'exploitation et l'injustice du système des castes. La prise de conscience politique des Intouchables et autres populations tribales, la tentation concomitante d'échapper (qui plus est collectivement) à leur statut d'infériorité en quittant l'hindouisme, alimentent chez les hindouistes le cauchemar d'une Inde dont la population défavorisée ne se dirait plus hindoue et ferait basculer l'équilibre religieux et démographique.
L'auteure analyse les conditions de conversion et démontre que le risque d'une hémorragie (ou de toute autre forme de sortie de l'hindouisme) reste limité, notamment du fait de la prégnance du système des castes. Le sous-titre de l'ouvrage, "Entre castes et églises ", pose ainsi l'un des enjeux centraux du christianisme indien : peut-on être chrétien et appartenir à une caste? Certains missionnaires ont souligné l'incompatibilité. Le système de castes fondé sur l'impureté contagieuse prescrit des règles d'évitement visant notamment la commensalité. Lorsqu'un converti de haute caste refuse de communier vu qu'à ses yeux, le chrétien de caste inférieure qui vient de boire le sang du Christ a souillé la coupe, on comprend la tentation des missionnaires d'extraire les convertis de la caste. D'autres missionnaires ont d'emblée compris qu'ils ne parviendraient à convertir qu'en acceptant le principe de la caste, et le jésuite Roberto Nobili alla jusqu’à déclarer publiquement : « La sainte loi spirituelle n'oblige personne à renoncer à sa caste [...] ni à faire quoi que ce soit de contraire à l'honneur de sa caste» (p. 144). Beaucoup se sont surtout trompés sur la signification de leur influence. Ainsi les femmes shanar du Sud de l'Inde qui, récemment converties, gagnent le droit de se couvrir la poitrine, signe d'un statut supérieur. Les missionnaires y voient la conversion au principe d'égalité ou aux vertus de la pudeur, ou un détournement de l'implacable hiérarchie des castes, là où l'auteure nous montre qu'il s'agit surtout d'une stratégie d'ascension sociale cohérente avec la logique de caste (adopter les coutumes des castes supérieures).

Catherine Clémentin-Ojha soutient que, dans l'Inde plurireligieuse, le système des castes prime sur l'identité religieuse, ce que confirme l'intégration historique des chrétiens indiens au monde de la caste. L'auteur souligne combien la caste repose sur des principes hindous. Mais, à défaut de porter crédit à l'idée hindoue selon laquelle le Christ serait, ici, une incarnation de Vishnu, on ne saurait dire jusqu'à quel point le chrétien-de-caste demeure 
nécessairement hindou. Devant la pauvreté des voix chrétiennes, on ne peut que s'associer à l'auteure pour souhaiter que la recherche indianiste apporte rapidement davantage de données ethnographiques contemporaines (à quoi ressemble précisément l'expérience religieuse et le quotidien des chrétiens indiens ?). Comme, à un autre niveau, les études de la diaspora nous ont appris qu'un hindouisme peut exister hors de l'Inde sans le système des castes, l'étude des chrétiens indiens pourrait bien éclairer d'un jour nouveau la sociologie religieuse de l'Inde, notamment en nous confrontant avec la complexité des interactions entre hindous et non-hindous, dans un contexte d'évolution des logiques croisées d'appartenances nationale, religieuse, et de caste.

Mathieu Claveyrolas

François Robinne \& Mandy Sadan, eds Social Dynamics in the Highlands of Southeast Asia. Reconsidering Political Systems of Highland Burma by E. R. Leach Leiden, Brill, 2007, 33I p., bibl., index, cartes (« Handbuch der Orientalistik, Section Three, Southeast Asia » 18).

$P$ Olitical Systems OF Highland BURMA d'Edmund R. Leach ${ }^{1}$ est l'un des ouvrages d'ethnologie qui suscita le plus d'engouement, de débats et de controverses. Depuis sa parution en 1954, des générations de chercheurs se sont positionnées par rapport aux conceptions iconoclastes du politique, de la culture, du rituel ou de l'ethnicité que Leach proposait dans ce livre, de même que fut amplement discuté le système relationnel correspondant au mariage avec la cousine croisée matrilatérale qu'il décrivait à travers l'exemple kachin. Rien d'étonnant donc à ce que François Robinne et Mandy Sadan, deux ethnographes contemporains des Kachin, aient organisé en 2004 un panel marquant le cinquantième anniversaire de la publication du livre de Leach, dans le cadre du congrès de la European Association of South-East Asian Studies (EUROSEAS), qui se tenait cette année-là à Paris.

Le présent ouvrage regroupe les contributions à ce panel, contributions que l'on peut classer en trois catégories reconnues in fine comme telles dans le plan du volume. Tout d'abord, une première catégorie d'articles introduit le livre de Leach par référence au contexte de l'époque et au curriculum de l'auteur. C'est le cas de ceux de Franck Lehman et de Robert Anderson. La longue introduction rédigée par Franck Lehman est remarquable à ce titre. Ce fin ethnographe des Chin, et l'un des premiers contradicteurs de Leach, y reprend les principales critiques adressées à l'auteur des Political Systems. Parmi ces critiques, il faut mentionner : la non-prise en compte des populations dites "kachin» qui vivent en Chine et ne se reconnaissent pas dans cet ethnonyme ; le rejet de l'analyse historique, sous l'influence du structuralo-fonctionnalisme de Radcliffe-Brown, qui conduit Leach à négliger divers facteurs externes ayant induit l'émergence de communautés " égalitaristes "; des approximations ethnographiques qui débouchent sur une typologie tronquée des formes politiques; ou encore le plaquage arbitraire du modèle

1. Edmund Ronald Leach, Political Systems of Highland Burma. A Study of Kachin Social Structure. Cambridge, Harvard University Press, 1954. 
oscillatoire de Pareto à des formes d'organisation complexes qui se développent sur un mode non pendulaire et échappent à la dualité simpliste gumsa/gumlao.

Les contributions de la seconde catégorie sont le fait d'ethnologues birmans ou chinois qui tirent parti de leur connaissance intime des groupes kachin pour relayer et approfondir certaines des critiques dont Franck Lehman se fait l'écho. Ainsi, sans remettre en cause fondamentalement l'opposition gumsalgumlao, Maran La Raw la nuance par renvoi à des catégories intermédiaires relatives au système hiérarchique gumsa que Leach avait ignorées, poussé qu'il était par le souci de conforter à tout prix ses hypothèses. Or, ces catégories sont des plus pertinentes pour interpréter les événements de 1939-1947 qui firent émerger un groupe kachin unitaire défendant la cause autonomiste face aux nationalistes birmans. Les remarques de Maran La Raw font également ressortir une approche très superficielle de la parenté de la part de Leach et un traitement de l'esclavage non moins problématique. Il fait de cet esclavage une nécessité structurale alors que celui-ci renvoie à des circonstances historiques bien précises. Mandy Sadan prolonge, de son côté, ces critiques en démontrant que la notion de gumlao ne devint saillante dans l'histoire des Kachin qu'avec l'avènement de l'ordre colonial britannique. Les leaders nationalistes kachin furent, dans ce contexte, prompts à exploiter la notion pour s'opposer à ce qu'ils percevaient comme une forme de despotisme étranger. Le reproche majeur que Mandy Sadan adresse à Leach est de réduire les catégories politiques kachin à des signifiés prétendument stables, alors que ceux-ci changent avec l'évolution de la société. Enfin, Ho Ts'ui-p’ing étaye considérablement la typologie kachin des formes de richesse que Leach avait esquissée. Selon l'ethnologue chinois, l'émergence d'un ordre social gumlao doit être interprétée par référence aux tensions entre richesses privatives et richesses "relationnelles » que le commerce de l'opium et la monétarisation de l'économie auraient engendrées.

La troisième catégorie de contributions à l'ouvrage est le fait d'une jeune génération d'émules de Leach. Ceux-ci partagent la conviction que, en dépit des multiples critiques formulées à l'encontre de l'auteur des Political Systems, ses propositions, concepts et modèles n'en demeurent pas moins utiles pour éclairer le fonctionnement des sociétés du Sud-Est asiatique. Selon Philippe Ramirez, il en irait ainsi de la fameuse proposition de Leach en vertu de laquelle seules les représentations forment des systèmes cohérents, les pratiques culturelles, simples "habits" des situations sociales étant conçues comme des accidents de l'histoire. Pourtant, les trois catégories ethniques du centre de l'Assam qu'il examine dans son article, celles des Dimasa, des Karbi et des Tiwa reposent sur un socle de pratiques culturelles pérennes qui invalident la proposition de Leach. D'où la conclusion embarrassée de Philippe Ramirez qui soutient que cette proposition reste effective mais doit être reformulée pour prendre en compte des contextes contemporains où la construction politique des identités révèle une plus forte dépendance entre culture et structures sociales (p. 107). Les tentatives consistant à interpréter l'organisation politique de divers groupes montagnards par référence au modèle gumsa/gumlao ne sont guère plus probantes. Pascal Bouchery avec l'étude des Naga et Vanina Bouté à propos des Phu Noi de Phongsali (Laos) aboutissent tous les deux à la conclusion que cette opposition pendulaire ne peut rendre compte du dynamisme des formes d'organisation politiques auxquelles ils s'intéressent, et que ces formes découlent non d'un déséquilibre structural, mais d'un faisceau de facteurs économiques, géographiques et historiques. Conclusion hautement prévisible si l'on considère avec Maran La Raw que le modèle gumsa/gumlao est une fiction théorique inventée par Leach pour les besoins de son argumentation structuraliste.

Plusieurs contributeurs au présent recueil reprennent également la notion de 
« langage rituel " par laquelle Leach rendait compte des codes partagés qui servaient tout à la fois de base de communication et de plateforme unitaire aux composantes ethniques hétérogènes de l'ensemble kachin. En soi, l'idée d'analyser les structures symboliques qui permettent aux groupes en contact de partager les mêmes règles du jeu et les mêmes enjeux est très féconde. C'est l'un des apports majeurs du livre de Leach, surtout si l'on considère qu'en son temps la thèse dominante était celle d'isolats ethniques se perpétuant sui generis. Chacun à leur manière, Stéphane Gros et François Robinne exploitent utilement cet aspect de la problématique des Political Systems. Le premier analyse les liens de dépendance fondés sur la notion de dette qui intègrent, par l'entremise de multiples actes symboliques, les Drung du NordYunnan dans un ordre hiérarchique supra-ethnique. Quant au second, il soutient que c'est le système d'échange matrimonial mayuldama et non l'opposition politique gumsa/gumlao qui sert de langage rituel commun aux groupes kachin. En témoigne, selon lui, le fait que des lignages de ces groupes se pensent équivalents et tenus les uns envers les autres par des règles de prohibition unitaires.

Si l'hypothèse de structures symboliques transethniques dégage des horizons de recherche prometteurs, le concept de « langage rituel » pose néanmoins problème, ainsi que le souligne Franck Lehman dans l'introduction. Concevoir les systèmes symboliques par renvoi aux règles de la linguistique est en effet assez simpliste. On touche ici à un défaut que partagent la plupart des émules de Leach ayant contribué au présent recueil. Ils reprennent les concepts qu'il a élaborés "clé en main", sans vraiment chercher à les dépasser pour reformuler ses intuitions novatrices en des termes plus opératoires. De surcroît, ils n'intègrent que très partiellement les riches discussions que ces concepts ont suscitées depuis cinquante ans. De manière générale, cet ouvrage révèle au grand jour l'improbable conciliation de deux approches du livre de Leach : l'une inductive, celle de Franck Lehman, Maran La Raw ou Mandy Sadan, qui ont renouvelé significativement l'ethnographie des Kachin mais accueillent avec scepticisme les épures théoriques plaquées sur des réalités sociohistoriques complexes; l'autre qui, à l'inverse, interprète quasi religieusement ces mêmes réalités à travers le prisme de l'approche hypothético-déductive du "maître à penser » britannique.

Bernard Formoso 
Arnaud Ruffier

Samarcande. Identités et espaces festifs en Ouzbékistan

Montreuil, Aux lieux d'être, 2007, 236 p., bibl., gloss.

LE TRAVAIL d'Arnaud Ruffier se propose d'analyser la société de Samarcande à travers ses pratiques festives. L'auteur part du constat que la société ouzbèke a vécu, au siècle dernier, des changements profonds : traditionnelle, elle est devenue soviétique, apparemment sans "trace de tribalisme, d'Islam, de féodalisme ou de capitalisme " (p. 10). Après les indépendances, se présente au visiteur une société qui semble garder à la fois un héritage soviétique et celui des années d'avant la révolution. Selon Arnaud Ruffier, ce sont surtout les fêtes qui permettent d'observer une juxtaposition de pratiques récentes et séculaires. L'étude de ces pratiques, se demande-t-il, nous aidet-elle à mieux cerner l'adaptation de la société au régime soviétique qui, par le biais de l'idéologie communiste, introduisait l'idée de nation et d'identité nationale commune à toutes les ethnies du pays? Les pratiques festives sont-elles " restées les reliquats folkloriques d'une organisation traditionnelle " ou bien constituent-elles " le reflet d'une organisation sociale que n'aurait pas totalement détruite la soviétisation » (p. 11) ? Il est tout à fait intéressant de vouloir analyser identités, fêtes et leurs enjeux sociaux, politiques dans un lieu délimité. Notamment, le rapport de la fête au "terroir ", à l'espace géographique et social que ce terme véhicule, peut être un sujet de recherche prometteur, d'autant plus que la fête ellemême provoque la création d'un espace : celui, "festif ", où se manifestent les identités des participants. Arnaud Ruffier aborde cette problématique sous deux angles: dans la première partie du livre, intitulée "Contexte sociopolitique et pratiques festives observées", il présente ses observations des pratiques festives « en contrepoint à la description des formes d'organisation sociale étudiées" (p. 14).
Cela doit permettre, selon lui, de situer la fête dans le politique, le social et l'histoire. Dans une deuxième partie, intitulée "Les fêtes ouzbèkes: un espace-temps de construction de la réalité sociale ", l'auteur étudie la fonction spécifique de chaque fête cherchant à décrire "le plus précisément possible certaines des logiques d'interactions mises en œuvre par les participants des fêtes " (id.). On trouve ici des descriptions des différents types d'espaces festifs ou de fêtes dans leur rapport à la société : to’y «la célébration d'un rite de passage ", ziyofat " un banquet", bayram "le jour de l'an ", ou gap " une fête d'amis ». L'auteur cherche dans ce contexte à retracer la construction d'une réalité sociale à partir de la réalité festive en recourant à des thèmes classiques d'anthropologie sociale: la naissance qui permet l'affirmation du statut de la mère, la circoncision qui marque le passage du garçon circoncis au groupe des hommes adultes, le don qui crée des alliances, etc. Sont aussi commentées certaines observations concernant la prise d'influence ou une sorte d'appropriation de la fête par la politique nationale qui « essaie de récupérer ces fêtes à son profit pour en faire l'expression pour partie d'une institutionnalisation des communautés territorialisées, de l'État et de la nation" (p. 206).

Tel est donc l'objectif du livre : étudier les «identités et espaces festifs en Ouzbékistan " comme l'indique le soustitre. Son titre, lui, semble plutôt éblouir qu'élucider : Samarcande, un nom qui renvoie à une ville lointaine, exotique pour certains, sur la route de la soie. Au cours de la lecture, on apprend que ce nom, désignant la ville et la région, délimite le champ d'étude. Mais peut-être cette petite confusion suscitée par les titres renvoie-t-elle à une problématique centrale déjà soulevée: 
dans quelle mesure l'idéologie soviétique a-t-elle su créer une société ouzbèke homogène et dans quelle mesure une "société samarcandoise » conserve-t-elle ses particularités culturelles?

À ce propos, l'auteur introduit son sujet par des observations sur l'identité dans le contexte samarcandois : une grande diversité d'ethnies habite la ville et sa région; on peut constater une «territorialisation " du lieu de séjour par rapport à l'appartenance ethnique ainsi qu'une "ethnisation" des rapports socio-économiques " (pp. 17 sq.), etc. Après cette introduction, le lecteur s'attend à une description d'espaces festifs tenant compte d'une multitude d'influences culturelles. Il n'en est rien, car Arnaud Ruffier prélève ses exemples uniquement dans les milieux ouzbèke et tadjik, sans suffisamment justifier ce choix. On pourrait croire, à lire l'auteur, que seuls ces deux groupes donnent à la fête son identité. Mais on peut se demander en quoi la diversité évoquée joue sur l'espace festif. Et cela nous ramène au titre : que désigne le mot Samarcande? Une ville et sa région? Une société samarcandoise homogène? Une multitude d'influences mentionnées, mais non étudiées?

Reste à commenter la nature du matériau collecté par l'auteur, qui lui fournit les bases de son analyse et qui devrait lui permettre d'illustrer ses remarques, car son terrain en Ouzbékistan lui a permis - on le suppose - de collecter un vaste corpus qui est sans nul doute d'un grand intérêt anthropologique. Pourtant, à cet égard, la récolte semble être étonnement maigre: Arnaud Ruffier se réfere, il est vrai, entre autres, à une interview (pp. 40-44), à ses observations de fêtes, etc. Mais ce matériau de première main déçoit car les informateurs restent étrangement absents de l'étude et les rencontres ethnologiquement intéressantes sont résumées d'une manière qui évoque plutôt les rapports de bureaucrates. Ainsi, l'interview n'est reproduite qu'en résumé et non dans les termes originaux et les descriptions des fêtes et autres événements sont schématiques et peu concrètes. L'analyse des espaces festifs devrait pourtant inclure celle de situations particulières, d'interactions ponctuelles entre invités (animosités, alliances...) ou de paroles prononcées. Depuis quels lieux, sinon à partir de ces faits situationnels et concrets, l'analyse anthropologique d'espaces festifs pourrait-elle se réaliser? 
E U R O P E

Marie-Claude Smouts, ed.

La situation postcoloniale. Les postcolonial studies dans le débat français

Paris, Presses de Sciences Po, 2007, 452 p., bibl., index.

\section{9} l'intérêt et les limites du postcolonial dans le débat français", écrit son éditrice, MarieClaude Smouts (p. 25). Ce recueil d'articles (et de discussions restituées dans une police de caractère de plus petite casse) est issu d'un colloque qui s'est tenu au CERI en mai 2006. Il est organisé en trois parties qui traitent respectivement des études littéraires comparées comme lieu d'émergence des postcolonial studies, de leur importation dans le champ des sciences sociales, et de l'application de leurs problématiques au cas de la France contemporaine. Sa richesse tient en particulier à la qualité et à la diversité des contributeurs, puisqu'il rassemble, d'une part, analyses franco-situées et écrivains ou auteurs postcoloniaux (Nuruddin Farah ou Akhil Gupta), d'autre part, spécialistes (Alexis Tadié ou Denis-Constant Martin, Jacques Pouchepadass, Jackie Assayag ou Romain Bertrand) et discutants plus éloignés de cette école (Georges Balandier ou Giovanni Levi). L'ensemble est précieux dans sa volonté de prendre au sérieux la production postcoloniale; il contient de très bons éléments de bibliographie et de critique, mais présuppose une connaissance préalable minimale du champ.

Pour le débat épistémologique en sciences sociales, la deuxième partie de l'ouvrage est indiscutablement la plus riche.
Disons toutefois rapidement que la problématisation de la première partie peut être résumée par les très belles phrases de l'écrivain Nuruddin Farah: "Je me suis demandé: que se passe-t-il quand un empire meurt? Qu'arrive-t-il à sa progéniture? Et j’ai pensé : de la beauté peut-elle naître de la mort, de la mort d'un empire? Une beauté, de nature différente bien sûr, peut-elle surgir des ruines d'un empire qui s'est effondré? " (p. 121). La présentation par Alexis Tadié du roman indien de langue anglaise, et l'analyse proposée par DenisConstant Martin de l'œuvre d'Édouard Glissant sont particulièrement riches dans ce contexte.

Tout en marquant l'intérêt du champ ouvert par les travaux postcoloniaux (et subalternistes) récents, une série convergente d'interventions (notamment celles de Jackie Assayag et de Romain Bertrand) discutent dans la deuxième partie de l'ouvrage un certain nombre de faiblesses méthodologiques de l'approche postcoloniale: le textualisme (et l'accent mis sur les représentations plutôt que sur les pratiques), le misérabilisme sociologique, la prétention de parler pour les dominés, le recours à des catégories chimériques, le manque d'enquêtes historiographiques. Cette démarche critique culmine dans l'article bref et incisif de Jean-François Bayart, qui écrit ainsi : 
«Les études postcoloniales sont à la fois utiles, superflues, assez pauvrement heuristiques et politiquement dangereuses" (p. 269).

L'article de Jacques Pouchepadass (pp. 173 sq.) donne un tableau plus nuancé, moins homogénéisant, et extrêmement riche du projet postcolonial (dans la même veine que dans son article "Les émeutes du "93" sont-elles postcoloniales?") ${ }^{1}$. Tout d'abord, il montre que l'opposition entre poststructuralisme et marxisme est une tension présente dès l'origine au sein des cultural studies. C'est le point de départ d'une critique empiriciste des études postcoloniales : «En ignorant superbement la spécificité des situations coloniales particulières, en faisant bon marché des tensions et des conflits de toutes sortes qui surgissaient sans cesse au sein même des élites dirigeantes chargées de la gestion des colonies, comme dans les métropoles à propos des colonies, en traitant en somme de la colonialité comme d'une configuration de pouvoir uniforme, invariante et continue, ce discours, inévitablement, se disqualifie aux yeux des historiens» (p. 191) Enfin, il constate que les critiques mentionnées ici reprennent avant tout des critiques internes: les postcolonialistes ont bien conscience qu'ils doivent enraciner leur discours critique dans l'histoire des pratiques effectives de domination et dans l'expérience vécue des subalternes.

Les aperçus proposés dans cet ouvrage permettent de revenir sur son titre; car de situation postcoloniale, il n'est en vérité question ici que de la seule France métropolitaine. La troisième partie tente en effet d'explorer dans le cas français l'héritage politique ou les traces plus vastes de l'expérience coloniale. On peut émettre quelques réserves sur le sens de la généalogie française des postcolonial studies mise en avant dans l'ouvrage: car s'il ne fait aucun doute que les propositions théoriques de Georges Balandier ont mis l'accent sur le poids de la situation coloniale sur les sociétés colonisées comme colonisatrices, les postcolonial studies ne se réduisent pas à cette idée, qui n'est pas au demeurant spécifiquement française (Max Gluckman en ayant fait la démonstration dix ans avant Balandier).

Par ailleurs, on est frappé de l'absence quasi totale de réflexions - à l'exception des remarques de Rada Ivekovic - sur la question de l'organisation de l'université et de la constitution des disciplines en France et aux États-Unis. Car les enjeux épistémologiques des études postcoloniales discutés dans l'ouvrage n'ont pu être débattus avec une telle vigueur qu'en raison de leur succès institutionnel outre-Atlantique.

À ces réserves minimes près, le recueil de Marie-Claude Smouts constitue un excellent complément aux ouvrages édités par Mamadou Diouf ${ }^{2}$ et par Neil Lazarus ${ }^{3}$.

\section{Michel Naepels}

1. Cette recension de l'ouvrage de Jean-Loup Amselle, L'Occident décroché. Enquête sur les postcolonialismes (Paris, Stock, 2008), est parue dans la rubrique "Débat » de L'Homme, 2008, 187-188: 413-422.

2. Cf. L'Historiographie indienne en débat. Colonialisme, nationalisme et sociétés postcoloniales, Paris, Karthala /Amsterdam, SePHIS, 1999.

3. Cf. Penser le postcolonial. Une introduction critique, Paris, Éd. Amsterdam, 2006. 


\section{Ethnologische Studien an europäischen und mediterranen Gesellschaften} Frankfurt-am-Main, Suhrkamp, 2008, 258 p., bibl., ill.

$S^{\prime}$ livre un panorama des recherches qu'il mène depuis de nombreuses années sur les sociétés méditerranéennes. Aussi son ouvrage, outre qu'il condense l'ensemble des théories et perspectives auxquelles ses analyses ont conduit, se présente-t-il sous la forme d'un plaidoyer pour une nouvelle approche globale en anthropologie.

Thomas Hauschild nous montre comment, après une phase marquée par les théories évolutionnistes, cette discipline qui s'est assignée le but de regarder au plus près du côté de l'étranger et de «l'étrange" a tenté d'analyser la culture par le biais d'approches relativistes. Les chercheurs croyaient pouvoir expliquer les différences culturelles par un multiculturalisme universaliste qui dissimulait mal leur eurocentrisme. La pensée politique européenne avait elle aussi besoin de comprendre des phénomènes apparemment inexplicables, tels que le "crime d'honneur" dans le milieu familial des migrants de l'espace méditerranéen. L'auteur dénonce une telle perspective qu'il considère comme réductrice. Selon lui, la théorie de la performance, qui s'est trop focalisée sur la structure des sociétés, devrait se compléter par un regard matérialiste, qui n'envisage pas les différences culturelles comme des entités fixes dans les structures sociales et avec lesquelles un individu devrait bricoler sa propre réalité souvent problématique, en particulier pour les immigrés venant de cultures extra-européennes.

Entre matérialisme et idéalisme, Thomas Hauschild milite ainsi pour un troisième regard, qui considère l'influence mutuelle entre la réalité matérielle et la culture appropriée aux acteurs sociaux. L'auteur, qui a collecté pendant plusieurs années de terrain des données sur le sud de l'Italie, établit des connexions entre le champ du religieux et le paysage, et regarde ainsi de plus près la constitution de la culture du sacré. Le développement des croyances populaires a mené aux trois grands monothéismes qui dominent aujourd'hui l'espace religieux méditerranéen. Selon Thomas Hauschild, s'appuyant ici sur la notion de bricolage telle que Fernand Braudel l'a utilisée, il existe une connexion étroite entre l'espace matériel et topographique et le religieux. Certes l'habitus et la mémoire participent de la constitution du sacré dans une société, mais l'environnement y joue aussi un rôle fondamental, la structure du paysage influençant fortement le fait social et culturel.

Thomas Hauschild poursuit ensuite sa réflexion par une analyse sur la Mafia. Il s'intéresse ici au regard scientifique sur cette organisation, regard longtemps empreint d'un certain romantisme, ainsi qu'en témoignent les travaux de chercheurs renommés tels que Jane Schneider et Anton Block. Pour Thomas Hauschild cependant, l'action de la Mafia en tant que système économique informel trouve davantage sa raison d'être dans la situation économique difficile des Siciliens qui n'a rien de "romantique». Il montre comment un tel système constitue de fait une véritable réserve d'action pour les régions pauvres et défavorisées d'Italie.

Puis, il continue plus en avant son investigation sur la violence en se penchant sur une autre échelle d'organisation, à travers l'étude d'Al-Qaida. Il fait ainsi un premier pas vers une analyse ethnologique d'AlQaida, permettant de pousser plus loin la réflexion conduite par certains chercheurs, tels que Hans Kippenberg et Navid 
Kermani, sur les actions du réseau, et d'aller au-delà des conclusions généralistes sur l'islam qui dominent aujourd'hui dans les médias.

Enfin, l'examen concret des faits de violence en milieu familial chez les immigrés originaires de pays islamiques et méditerranéens en Allemagne révèle, à l'instar du regard porté sur la Mafia, que les individus coupables de ces faits sont souvent considérés, jusque devant les tribunaux, comme victimes de leur propre mentalité. L'auteur plaide pour un point de vue qui ne se fonde pas uniquement sur le mentalisme culturel, en constatant qu'un code fixe de l'honneur n'existe pas, qu'il s'agit d'un système social dont l'apparence varie de société à société. En revanche, comme son analyse le révèle, les personnes jugées pour des faits de violence viennent souvent d'un milieu pauvre et semblent manquer de ressources tant sociales qu'économiques et culturelles.

En conclusion de son ouvrage, Thomas Hauschild argumente donc pour une approche plus matérialiste sur les faits sociaux et culturels, qui combinerait les concepts de l'habitus bourdieusien et de la mémoire chez Maurice Halbwachs et Jan Assmann avec l'approche de l'héritage marxiste.

Par la richesse des données et la fécondité des perspectives de recherche auxquelles il convie, ce livre est une contribution essentielle à l'anthropologie de la Méditerranée. Il n'est pas seulement un résumé des recherches en cours sur les rites et la violence dans cette région, mais propose en même temps un regard sur la situation difficile d'une discipline universitaire dans un pays européen comme l'Allemagne. Une discipline qui a la lourde responsabilité d'expliquer des faits sociaux et culturels, leurs circonstances et leurs causes. Thomas Hauschild souligne qu'une expertise locale et de longue durée est nécessaire. Si la recherche anthropologique ne peut pas résoudre les problèmes de nos sociétés, elle peut néanmoins indiquer des chemins et apporter des clés essentielles pour comprendre les mutations et les dynamiques sociales du monde contemporain.

Barbara Peveling 


\section{OCÉ A N I E}

\section{Stuart Kirsch \\ Reverse Anthropology. \\ Indigenous Analysis of Social and Environmental Relations in New Guinea Stanford, Stanford University Press, 2006, 272 p., bibl., index, ill., fig. cartes.}

C

E LIVRE se focalise sur les luttes politiques de deux peuples établis des deux côtés de la frontière séparant la Papouasie Nouvelle-Guinée et le West Papua ${ }^{1}$ : les Yonggom et les Muyu, qui parlent des dialectes d'une même langue non austronésienne et partagent nombre de relations et de traits sociaux et culturels. L'un de ces combats porte sur les dévastations de l'environnement résultant de l'activité de la mine de cuivre et d'or de Ok Tedi en Papouasie Nouvelle-Guinée, l'autre concerne quelque 6000 réfugiés de West Papua qui, depuis la Papouasie NouvelleGuinée, militent pour l'indépendance de leur pays d'origine. À la demande des Yonggom, l'anthropologue américain Stuart Kirsch a collaboré sur des questions d'environnement avec des avocats et des organisations non gouvernementales (ONG). Cette expérience l'a conduit à écrire ce livre en lui donnant "l'occasion d'observer comment les analyses indigènes offrent des interprétations alternatives de relations politiques et d'événements historiques dont la valeur a été avérée dans divers contextes» (p. 3). Pour Stuart Kirsch, il s'agit d'une pratique originale de l'anthropologie qu'il qualifie d'anthropologie inverse et qui aide "à la reconnaissance de la critique indigène et à l'articulation d'alternatives politiques, alignant ainsi temporairement l'ethnographie sur les objectifs des mouvements sociaux" (ibid.). Il entend mettre au premier plan les représentations indigènes des événements, évitant de "privilégier d'autres cadres de référence " (ibid.). Cependant, cette insistance sur l'aspect emic des représentations et moyens de lutte adoptés doit être relativisée du fait de l'appui et de l'intervention des ONG. Ainsi, la reproduction d'une carte postale éditée par l'une d'entre elles, le Mineral Policy Institute, représentant un collage du fleuve Ok Tedi dans le site du port de Sydney (pp. 21-22), évoque irrésistiblement le concept situationniste de "détournement" ou les collages politiques de John Heartfield.

Le premier chapitre décrit les rencontres historiques qui ont précédé la période actuelle. L'auteur évoque successivement les chasseurs d'oiseaux de paradis, les collectionneurs occidentaux de cuirasses tressées et de têtes humaines (les Yonggom sont les infortunés voisins de peuples marind), mais aussi certains incidents ayant marqué les premiers contacts avec des administrateurs coloniaux de brousse, les effets de la Seconde Guerre mondiale, ceux de l'indépendance de la Papouasie Nouvelle-Guinée

1. Aussi connu comme Irian Jaya, la partie ouest de la grande île, colonisée par l'Indonésie. 
et, plus récemment, une révolte d'ouvriers employés dans un forage. Les chapitres suivants (II à IV) présentent les points de vue yonggom sur le monde dans la mesure où ils sous-tendent la résistance de ce peuple aux compagnies minières. En particulier, Stuart Kirsch « traite du rituel et de la magie comme de modes d'analyse [...], examine ce qu'ils nous révèlent sur le monde" (p. 76), en soulignant le rôle des échanges. L’originalité du livre, suivant en cela Nancy D. Munn ${ }^{2}$, est de s'intéresser de près à la création de la «valeur négative " que représente l'échec des échanges, en particulier autour de la non-réciprocité. Dans cette veine, l'auteur interprète l'apparition de "cultes-cargo", dans les années 1950, comme résultant de situations d'échecs dans la réciprocité d'échanges matrimoniaux et funéraires régionaux, impliquant des Yonggom, des Muyu et d'autres peuples. L'association de cultes-cargo à un déni de réciprocité n'est pas nouvelle, ce qui l'est c'est qu'ici la non-réciprocité est envisagée entre peuples voisins et pas avec les colonisateurs ou évangélisateurs. Toutefois, sur un plan théorique, il me semble regrettable que ne soient pas confrontées à celles défendues ici les thèses d'Annette B. Weiner $^{3}$, qui mettent précisément en question la "réciprocité comme norme». Cette première moitié du livre correspond ainsi à une mise en place sérieusement argumentée de conceptions muyu et yonggom, qui fonde les propositions de la seconde partie.

Les analyses consacrées à la mine et aux revendications indépendantistes s'ouvrent sur le thème de la sorcellerie, dont le discours - avec ceux concernant le diable et la magie - sont « des ressources de grande valeur pour de nombreuses sociétés quand il s'agit d'analyser les contradictions du capitalisme, l'exercice du pouvoir étatique et les problèmes de la modernité [...]. Les Yonggom invoquent eux aussi le discours de la sorcellerie en relation à la mine d'Ok Tedi, comparant la compagnie minière et son impact sur l'environnement aux conséquences nuisibles de la sorcellerie. Ils envisagent la compagnie minière comme un individu (corporate individual) et l'accusent d'agir de façon irresponsable - comme un sorcier. La mine les a obligés à "vivre dans la peur" en raison des risques inconnus associés aux [...] déchets rejetés dans leur système hydrologique. [La peur était un] sentiment auparavant associé à la crainte des représailles après un meurtre par sorcellerie. À travers leur interprétation de maladies, blessures et accidents qui auparavant auraient été attribués à la sorcellerie, les Yonggom manifestent l'analogie entre sorcellerie et exploitation minière en termes d'impact destructeur" (pp. 107-108), des destructions qui mettent en cause les conditions mêmes de leur existence. Perçue comme intentionnelle, la sorcellerie fait émerger des émotions d'ordinaire retenues : sentiments de perte, douleur, peur, colère, honte. Alors que « les Euro-Américains peuvent expliquer ces problèmes en termes environnementaux ou en se référant à l'économie politique de la globalisation, [...] les Yonggom, eux, utilisent le discours sur la sorcellerie pour souligner l'économie morale des relations sociales" (pp. 120126). Et les exemples donnés par Stuart Kirsch ont en effet de quoi surprendre les responsables des entreprises pollueuses mais aussi certains responsables d'ONG.

L'analyse des demandes de compensations des Yonggom pour les dégâts miniers est d'un grand intérêt. Ces demandes sont à la fois l'expression d'une tendance internationale ${ }^{4}$ et une démonstration de la façon

2. Cf. The Fame of Gawa. A Symbolic Study of Value Transformation in a Massim (Papua New Guinea) Society, Cambridge-New York, Cambridge University Press, 1986.

3. Cf. Inalienable Possessions. The Paradox of Keeping-While-Giving, Berkeley, University of California Press, 1992.

4. Elle est à l'œuvre par exemple en NouvelleCalédonie, mais depuis quelques années seulement, où l'aspect juridique de la revendication kanak commence à se développer, autour de questions relatives aux ressources minières et à l'environnement. Cette troisième phase de revendication vient après une première, plus particulièrement politique, bien connue, qui fut suivie (mais pas remplacée) par une seconde mettant l'accent sur ... /... 
dont le concept mélanésien de compensation s'est "déployé avec un grand potentiel créatif»(p. 128). L'auteur s'appuie ici sur des propositions théoriques de Marilyn Strathern ${ }^{5}$. Il montre aussi comment, privées de la médiation étatique en raison de la domination néo-libérale s'étendant à toute la planète, ces demandes s'orientent directement vers les entreprises. Elles ne s'indexent pas sur de la valeur marchande, mais sur des relations sociales: celles-ci peuvent varier d'une hostilité ouverte jusqu'à un refus d'accès à la ressource. Les recherches de Bruno Latour et Arturo Escobar sont à leur tour consultées pour souligner que, dans le cas de dégâts environnementaux, "quand le périmètre de la recherche est limité aux sciences naturelles, des problèmes qui sont de caractère hybride et combinent des personnes, des choses et des idées en un ensemble unique de relations ne peuvent être analysés de façon adéquate» (p. 129).

L'autre combat politique présenté par Stuart Kirsch dans le cadre de cette anthropologie inverse est celui des Muyu de West Papua, réfugiés politiques en Papouasie Nouvelle-Guinée. Eux aussi mobilisent des aspects de leurs vision et emprise traditionnelles sur le monde pour rendre compte de leur condition. Ils analysent leur situation politique complexe en termes de ses conséquences immédiates pour les relations sociales à travers mythe, histoire et divination. D'abord soupçonné d'être un espion, l'anthropologue deviendra ensuite leur témoin et intercesseur - comme souvent aujourd'hui et dans le passé pour d'autres peuples (chap. V et VI). Le septième chapitre signale un retour aux Yonggom et à la mine, dans le cadre de perspectives d'avenir. La conclusion reprend la question de la place de « l'analyse indigène » dans les interprétations, réactions et actions politiques, ainsi que sur les enseignements que nous pouvons en tirer. Ces "modes d'analyse peuvent fournir des stratégies de riposte que les Yonggom trouvent productives et par conséquent suggèrent d'autres façons de penser ces questions» (p. 217).
Pour la notion d'anthropologie inverse, dans laquelle les peuples qui nous intéressent proposent leur propre analyse de notre monde, James Clifford et, surtout, Roy Wagner $^{6}$, sont les références intellectuelles de Stuart Kirsch. De ce côté-ci de l'Atlantique, dès le début des années 1970, Robert Jaulin avec une partie des enseignants-chercheurs du (défunt) département d'ethnologie de l'Université Paris VII avaient fait des propositions analogues, dans une visée d'ethnologie impliquée. Les rôles pionniers de Clifford et Wagner ainsi que la prétention de Kirsch à une " approche nouvelle de l'étude des mouvements indigènes " (p. 3) doivent donc être relativisés du fait de l'ignorance des recherches non anglophones qui caractérise de nombreux collègues anglo-saxons ${ }^{7}$. Avec Reverse anthropology, l'auteur présente une réalisation, certes partielle, de ces perspectives théoriques en une analyse précise, bien documentée et argumentée, d'interactions entre des peuples de Mélanésie, des institutions étatiques, des ONG et des entreprises capitalistes

[Suite de la note 4] "la culture, la coutume" (cf. Denis Monnerie, La Parole de notre maison. Discours et cérémonies kanak aujourd'hui, NouvelleCalédonie, Paris, CNRS Éd.-Éd. de la Maison des sciences de l'homme, 2005). Celle-ci a été négligée par de nombreux observateurs. Aujourd'hui, ces trois domaines - "politique ", "culturel/coutumier" " juridique" - sont constitutifs de la revendication kanak dans son ensemble. Dans ce cadre, de façon caractéristique, les mêmes personnes agissent souvent dans ces trois domaines, tout en les distinguant en fonction de relations et contextes sociaux différents, ce qui rejoint certaines des analyses de Stuart Kirsch et de ses inspirateurs concernant la Mélanésie - et contribue à désarçonner les observateurs occidentaux.

5. Cf. Property, Substance and Effect. Anthropological Essays on Persons and Things, New Brunswick, Athlone Press, 1999.

6. Cf. The Invention of Culture, Chicago, University of Chicago Press, 1981.

7. Parfois aussi il s'agit de l'utilisation de travaux de chercheurs non anglophones qui peut se présenter comme originale car elle est faite sans références. Cette question est délicate, puisqu' elle touche aussi aux conversations informelles entre collègues et à l'air du temps que respirent les chercheurs du monde entier. 
transnationales minières. Ailleurs en Nouvelle-Guinée (Bougainville et West

Papua) et en Nouvelle-Calédonie, d'autres mines ont provoqué et provoquent encore d'autres réactions de la part des peuples autochtones. En Polynésie, le cas du ravage de Nauru par l'exploitation inconsidérée de ses mines de phosphate est bien connu. À la prospérité, au gaspillage (et aux escroqueries), sans pareils pendant quelques années, ont succédé, avec l'épuisement de cette ressource, des conditions de vie désormais difficiles pour les gens de Nauru. Les réactions, la résistance, les revendications des peuples autochtones face à l'exploitation de leurs ressources minières ne sont pas toutes, loin de là, similaires. Elles peuvent, ne l'oublions pas, comme dans le cas de la mine de Panguna à Bougainville, prendre des formes d'une extrême violence sur le long terme. Les spécialistes des sciences politiques, avec leurs discours et concepts universaux - certains diraient passe-partout -, semblent actuellement avoir le monopole de la réflexion sur ces phénomènes. Toutefois, les travaux anthropologiques concernant les impacts des mines dans le Pacifique commencent à se développer ${ }^{8}$ : tel est le cas de ce livre exigeant ${ }^{9}$ et au plus près du point de vue mélanésien des Yonggom et des Muyu, au moyen duquel Stuart Kirsch contribue au développement d'une anthropologie comparatiste du pillage des ressources et des ravages de l'écologie causés par les mines dans cette partie du monde. De tels fondements scientifiques me semblent indispensables à toute démarche sérieuse et efficace d'anthropologie impliquée. En retour, ce type de recherches - débutées en 1987 dans le cas de Kirsch pose la question de financements à long terme de travaux pouvant demeurer vraiment indépendants des ONG qui, le plus souvent, organisent les revendications, et, surtout, libres des influences des forces coloniales, néo-coloniales et capitalistes qui soutiennent et/ou contrôlent largement les États et industries, en Mélanésie et ailleurs.

Denis Monnerie

8. Par exemple pour la mine d'Ok Tedi : cf. Glenn Banks \& Chris Ballard, eds, The Ok Tedi Settlement. Issues, Outcomes and Implications (Canberra, National Centre for Development Studies, Research School of Pacific Studies, Australian National University, 1997) et l'article de Tony Crook, " "If You Don't Believe our Story, At Least Give Us Half of the Money" : Claiming Ownership of the Ok Tedi Mine, Papouasie Nouvelle-Guinée " (Journal de la Société des Océanistes, 2007, 125 : 221-228), qui fournit un intéressant contrepoint au livre de Stuart Kirsch. Pour une perspective plus large, cf. Glenn Banks \& Chris Ballard, « Resource Wars: The Anthropology of Mining", Annual Review of Anthropology, 2003, $32: 287-313$.

9. On relève toutefois quelques problèmes de pagination dans l'index. 